\title{
Systematic review of diabetes management among black African immigrants, white and South Asian populations
}

\author{
Folashade T Alloh', Ann Hemingway ${ }^{1}$, Angela Turner-Wilson ${ }^{1}$ \\ ${ }^{1}$ Bournemouth University, Faculty of Health and Social Science, United Kingdom \\ Keywords: global health \\ https://doi.org/10.29392/joghr.3.e2019020
}

\section{Journal of Global Health Reports}

Vol. 3, 2019

\begin{abstract}
Background
This study aims to explore the differences in the management of diabetes outcomes and prevalence among black Africans, white and South Asian populations living in western countries from published evidence. This review incorporates findings from differences in diabetes management outcome among black Africans compared to white and South Asian populations.
\end{abstract}

\section{Methods}

A systematic search of major electronic databases with peer review publications was conducted. PubMed, CIHNAL, Medline, Web of Science, Scopus, and Science direct databases were searched from 2007-2018. Relevant journals and citations from references were searched for selection in the review. Data were analysed to understand differences in diabetes outcomes among these populations.

\section{Results}

Fifteen articles met the inclusion criteria out of the sixty-six articles retrieved and included in the review. Majority of the articles were cross-sectional quantitative studies $(n=10)$ and qualitative studies $(n=5)$. Diabetes prevalence and outcome measures such as haemoglobin A1c, blood pressure, cholesterol and body mass index were reported to be higher among black African than white populations. The data showed disparity in diabetes management among black Africans as compared to white and South Asian groups.

\section{Conclusions}

The poorer health outcomes reported among black Africans as compared to white and South Asian populations suggest poor diabetes management. Further research is needed to understand why there is such disparity in the health outcome of black African populations living with diabetes in western countries. There is a need to have a consistent target outcome measure in studies. Further synthesis was not possible due to differences in outcome measures used by studies reviewed.

The World Health Organization (WHO) reported diabetes to be among the ten leading causes of death in the last decade 2002-2012. ${ }^{1}$ Diabetes is estimated to affect approximately 422 million people globally. ${ }^{2}$ This has been estimated to increase to over 642 million in 2040. ${ }^{3-5}$ Overall, there has been an increasing trend in diabetes cases globally.

Diabetes has constantly been a public health burden in western countries such as the United Kingdom (UK), United States of America (USA) and Canada. ${ }^{6}$ Specifically, in the $\mathrm{UK}$, diabetes prevalence is put at $6.2 \%$ equivalent to more than 4 million people affected by the condition. ${ }^{7}$ In the USA, diabetes affects more than 30 million people (prevalence $9.4 \%) .{ }^{8}$ Canada has $9.2 \%$ prevalence, translating to 3.5 million people. ${ }^{9,10}$ This global public health issue requires further research into prevention and management of subsets of the population.

However, diabetes burden is higher among immigrant groups that make up ethnic minority groups in western countries. ${ }^{11,12}$ According to the National Institute for Health and Care Excellence (NICE) ethnicity is a risk factor for the development of type 2 diabetes. ${ }^{13}$ Ethnicity is defined as the collection of individuals that can be grouped together as a result of shared national or cultural values, which can include religion, language, ancestry and society. ${ }^{14}$ Ethnic minority groups are individuals that belong to groups and have different cultural traditions from the main population. ${ }^{15}$ black African group is a minority group that are particularly affected by diabetes. They have been reported to have higher prevalence of diabetes in the litera- 
Table 1. Search terms with synonyms

\begin{tabular}{ll}
\hline $\begin{array}{l}\text { Search } \\
\text { terms }\end{array}$ & \\
\hline $\begin{array}{l}\text { Diabetes } \\
\text { (S1) }\end{array}$ & "Diabet*" OR "Non-Insulin depend*" OR "Insulin resist*" OR "impair* glucose toleran*" OR "Dm2" OR "NIDDM" \\
$\begin{array}{l}\text { African (S2) } \\
\text { Othnic } \\
\text { minority (S3) }\end{array}$ & "Africa*" OR "black*" OR "Race* OR Afro* OR Afric* \\
$\begin{array}{l}\text { Management } \\
\text { (S4) }\end{array}$ & BME OR Ethnic* OR Minorit* OR "Ethnic* Minorit*" OR migrant* OR immigrant* \\
$\begin{array}{l}\text { Search } \\
\text { combination }\end{array}$ & S1 AND S2 AND S3 AND S4 \\
\hline
\end{tabular}

Table 2. Literature inclusion and exclusion criteria

\begin{tabular}{ll}
\hline Inclusion criteria & Exclusion criteria \\
\hline Online articles & Review papers \\
Primary research & Not peer reviewed \\
Peer-reviewed & Published before 2006 \\
$\begin{array}{l}\text { Published between 2006-2018 } \\
\text { English written }\end{array}$ & Other language outside English \\
$\begin{array}{l}\text { Considers Type 2 diabetes in black Africans } \\
\text { Compares diabetes management among ethnic groups }\end{array}$ & Considers other diseases in black Africans \\
$\begin{array}{l}\text { Measures diabetes management outcomes } \\
\text { Primary research: Qualitative, quantitative and mixed } \\
\text { method studies. }\end{array}$ & No reported ethnic group measures \\
\hline & Report other outcomes aside diabetes management \\
\hline
\end{tabular}

ture. ${ }^{16,17}$ For instance, black Africans in Netherlands were reported to be more than five times more at risk of developing diabetes than the general population. ${ }^{18}$ Similarly, studies have reported health disparities among ethnic minorities as compared to the general population in the USA with reported health inequalities among these groups. ${ }^{19} \mathrm{Di}-$ abetes prevalence among African immigrants has been reported to increase with years of migration. ${ }^{20}$ This presents the need to understand disparity in health by comparing diabetes outcome measures among black Africans to white and South Asian populations.

The aim of this review is to explore the differences in diabetes management outcomes among ethnic groups with focus on black African population's management. The interest in black African immigrants is because of the growth in this population and also due to the disparity in diabetes prevalence among this group compared to the general population in the host country. For example, they are among the highest growing ethnic minority groups in the UK. ${ }^{21,22}$ Although reviews have been conducted on diabetes management among South Asian and Caribbean groups 23-25, not much have been done in respect to black African population. To our knowledge, this is the first review comparing diabetes management outcomes among black Africans, white and South Asian populations.

\section{METHODS}

\section{STUDY DESIGN AND DATA SOURCES}

This review was carried out based on Search, AppraisaL, Synthesis and Analysis (SALSA) systematic review framework. ${ }^{26-28}$ Major databases were searched including: PubMed, CIHNAL, Medline, Web of Science, Scopus, and Science Direct were searched because they offer a good selection of publications that are related to this review question. The main search terms for this work were 'Diabetes', 'Africa', 'ethnic minority', 'management' and their alternative synonyms were entered into the databases searched (Table 1). These search terms were combined using Boolean operators to allow full retrieval of all articles that are related to the focus of this review. The protocol for this review has been registered on PROSPERO (CRD 42018088311).

\section{SELECTION CRITERIA}

To meet the inclusion criteria, articles that are reported to be primary research, use either qualitative or quantitative methodology and peer-reviewed were selected for this review. The results of the search were screened from the title, abstract and finally full text. The First author (FA) screened all article's title and abstract to assess their eligibility as meeting the inclusion criteria for the review. Details of inclusion and exclusion criteria are presented in Table 2 . 


\section{ASSESSING QUALITY OF THE PAPERS}

Each paper was appraised to assess the quality using relevant Critical Appraisal Skills Programme CASP ${ }^{29}$ checklist for cross-sectional quantitative studies and another for qualitative studies was used for the appraisal. This checklist was selected as it provides a structural approach to assessing rigour of the articles. ${ }^{30,31}$ A score was assigned to each article based on how the study answered screening questions giving No $=0$ and Yes $=1 .{ }^{32}$ Each study score was converted to the percentage of the total numbers of questions. It was decided to exclude any study that score below $50 \%$, this was to avoid reviewing papers with less quality. No study had $100 \%$ of the screening score and no study was below $50 \%$ and so no study was excluded based on quality assessment as they were of satisfactory quality. Data were extracted from these studies to be included in the review.

\section{DATA EXTRACTION}

Following the quality assessment of each paper, data were extracted using a data extraction tool. There are various types of data extraction tools that can be used in a review including paper, spreadsheets, email and web-based survey. The type used depends on the availability of funding, time for review and number of reviewers on the project. ${ }^{33}$ Data was extracted using Microsoft Excel (Microsoft Inc., Seattle WA, USA) spreadsheet by FA. Data were extracted on the aim of the study, the type of research methodology used, demographic information of the participants, key findings and conclusions from the study (Table 4).

\section{DIABETES OUTCOME MEASURE TARGETS}

In the quantitative articles that were included in the review, diabetes prevalence and four outcomes measures were reported in most studies reviewed (haemoglobin a1c level, blood pressure, cholesterol level and body mass index). These outcomes were selected as they were reported by most of the articles reviewed. In addition, these outcomes are used as the most accurate measure of diabetes management and as an indicator of biomedical outcomes of care. ${ }^{49,50}$ Other measures like smoking, awareness, knowledge, self-management and complications were reported in some studies but are not reported in this review. Different outcome measures were used in the studies reviewed; this was mainly based on the geographical location of the study. For example, studies conducted in North American mainly use American Diabetes Association (ADA) and International Diabetes Federation (IDF) target recommendations. Studies conducted in the UK use NICE target recommendations while studies in Europe use WHO target recommendations. It was not possible to conduct a metaanalysis on the articles due to the heterogeneity of the data from the studies reviewed, particularly from target outcome measures used (Table 3).

\section{DATA ANALYSIS}

Understandings of ethnicity within this literature review were based on the original authors' concepts.
The term "Whites" was used to refer to people from the European or Caucasian origin. South Asia included people originating from Indian, Pakistan, Bangladesh, Maldives and Sri Lanka, while Black population was used to refer to people originating from Africa and the Caribbean. Studies review reported establishing ethnicity based on self-reported origin, country of birth or as indicated in the hospital records in the studies reviewed.

Articles reported recruiting black populations with no further clarification on the specific black group. Further information on specific group of black population recruited for study is needed due to several groups that make up the black population. This presented difficulty as it was not made clear if recruited African immigrants that migrated from Africa or African Caribbean that migrated from Caribbean countries. This is due to the cultural and social differences between the two groups, such as language, diet, religion, geography, acculturation and socioeconomic factors, combining these groups as one ethnic group does not allow imprecision and probably unreliable. Only two studies reported findings between Black African and African Caribbean in separate categories. ${ }^{34,46}$ This highlights the need to understand the heterogeneity Black population.

\section{RESULTS}

\section{SEARCH RESULTS}

Articles published after 2006 were retrieved, this is to allow retrieval of more recent and current studies to be included in the review. Hard copies of all 66 full-text articles were printed out for a full screening; this led to the selection of 15 articles in the review with five qualitative and ten quantitative articles. A PRISMA flow diagram (Figure 1) was used to present details of the literature search and article retrieval. ${ }^{59}$ Full articles were printed to allow unbiased selection of articles to be included in the review and note taking on each article during appraisal.

\section{STUDY CHARACTERISTICS}

The ten quantitative studies included in this review were all cross-sectional studies. Sample size ranged from 359 to 34,345 with 127,273 total participants from all ten studies. All studies recruited participants aged 18 years and above. All the studies involved both male and female participants. Twelve studies were conducted in Europe with nine quantitative studies $34-36,40-42,45,47,60$ and three qualitative studies. ${ }^{39,44,48}$ Three studies were conducted in American with quantitative study ${ }^{43}$ and two qualitative 37,38 studies. Characteristics and key findings of each study are presented in Table 4.

\section{HETEROGENEITY OF BLACK POPULATION}

The heterogeneity of black population globally has necessitated the need for definition of specific groups of people in the black population. This is because of the sensitivity of findings from healthcare research requires focus on the specific group of blacks recruited in studies which may not be valuable if different groups of blacks are grouped as one. Therefore, there is a need to define subset groups within 
Table 4. Demographic of participants in studies review with key findings and conclusion

\begin{tabular}{|c|c|c|c|c|c|c|}
\hline Study & Aim & Study design & $\begin{array}{l}\text { Study } \\
\text { quality }\end{array}$ & $\begin{array}{l}\text { Participant's } \\
\text { demography }\end{array}$ & Key findings & Conclusion \\
\hline $\begin{array}{l}\text { Ballotari } \\
\text { et al. } \\
2015^{34}\end{array}$ & $\begin{array}{l}\text { To compare } \\
\text { prevalence of } \\
\text { diabetes } \\
\text { among } \\
\text { immigrants and } \\
\text { Italians and to } \\
\text { evaluate the } \\
\text { disparities in } \\
\text { the } \\
\text { management } \\
\text { and glycaemic } \\
\text { control. }\end{array}$ & $\begin{array}{l}\text { Quantitative } \\
\text { approach } \\
\text { measuring } \\
\text { clinical } \\
\text { outcomes of } \\
\text { prevalence } \\
\text { and HbA1C } \\
\text { value }\end{array}$ & $\begin{array}{l}17 / 20 \\
85 \%\end{array}$ & $\begin{array}{l}\text { Citizenship: } \\
\text { High } \\
\text { Developed } \\
\text { Countries } \\
\text { (HDC) High } \\
\text { Migration } \\
\text { Countries } \\
\text { (HMC) And } \\
\text { High } \\
\text { Migration } \\
\text { Pressure } \\
\text { Countries } \\
\text { (HMPC). } \\
\text { Sampling: } \\
\text { Population- } \\
\text { based data } \\
\text { Sample size: } \\
\text { 17,195. } \\
\text { 15,889 Italian, } \\
\text { 11 HDC, 1,295 } \\
\text { HMPC. Age } \\
\text { Range: 20-74. } \\
\text { Gender: Male } \\
\text { and Female. } \\
\text { Context: Italy }\end{array}$ & $\begin{array}{l}\text { Foreigners } \\
\text { younger with } \\
\text { type } 2 \text { diabetes } \\
\text { than Italians } \\
\text { Sub-Saharan } \\
\text { Africans with } \\
\text { lowest mean age } \\
44.6 \text { years for } \\
\text { Female and } \\
44.9 \text { years for } \\
\text { Male. Both sexes } \\
\text { experience a } \\
\text { higher } \\
\text { prevalence of } \\
\text { diabetes than } \\
\text { Italian. Lower } \\
\text { Italians not } \\
\text { performing } \\
\text { HbA1C } \\
\text { compared to } \\
\text { immigrants. } \\
\text { Immigrants had } \\
\text { worse indicator } \\
\text { in HbA1C } \\
\text { measure. Higher } \\
\text { odds of not } \\
\text { being tested for } \\
\text { HbA1C than } \\
\text { Italians. } \\
\text { Immigrants } \\
\text { experience } \\
\text { higher odds } \\
\text { compared to } \\
\text { Italians for not } \\
\text { being in the care } \\
\text { of diabetes } \\
\text { clinics, without } \\
\text { HbA1C tests in } \\
2010 \text { and with } \\
\text { HbA1C >=9\%. }\end{array}$ & $\begin{array}{l}\text { Confirmation of } \\
\text { the higher } \\
\text { prevalence of } \\
\text { diabetes among } \\
\text { immigrants than } \\
\text { Italian. } \\
\text { Immigrants are } \\
\text { less compliant and } \\
\text { more likely to } \\
\text { experience worse } \\
\text { levels of HbA1C }\end{array}$ \\
\hline $\begin{array}{l}\text { Dreyer et } \\
\text { al. } 2009^{35}\end{array}$ & $\begin{array}{l}\text { To establish the } \\
\text { impact of } \\
\text { ethnicity on } \\
\text { the prevalence } \\
\text { and severity of } \\
\text { diabetes } \\
\text { mellitus and } \\
\text { Chronic Kidney } \\
\text { Disease (CKD) }\end{array}$ & $\begin{array}{l}\text { Quantitative } \\
\text { Cross- } \\
\text { sectional } \\
\text { study }\end{array}$ & $\begin{array}{l}17 / 20 \\
85 \%\end{array}$ & $\begin{array}{l}\text { 34,359 Adults } \\
\text { coded white } \\
\text { black and } \\
\text { South Asia } \\
\text { population }\end{array}$ & $\begin{array}{l}\text { Overall } \\
\text { prevalence for } \\
\text { white } 3.5 \%, 11 \% \\
\text { for South Asian } \\
\text { and } 8 \% \text { for black } \\
\text { population. } \\
\text { Diabetic } \\
\text { proteinuria was } \\
\text { more frequent } \\
\text { among Blacks } \\
\text { compared to } \\
\text { white group } \\
\text { (22.4\% vs. } \\
14.1 \% \text { ) but } \\
\text { similar in South } \\
\text { Asia ( } 21 \% \text { Vs } \\
22 \%) \text {. Lower } \\
\text { CKD when } \\
\text { compared with } \\
\text { white } \\
\text { population. } \\
\text { Blood pressure } \\
\text { is less controlled } \\
\text { in black group } \\
\text { regardless of } \\
\text { CKD, less than } \\
50 \% \text { of diabetics } \\
\text { achieve Bp = }\end{array}$ & $\begin{array}{l}\text { Higher level of } \\
\text { diabetes among } \\
\text { ethnic minority } \\
\text { groups was } \\
\text { supported with } \\
\text { this study. Black } \\
\text { population have } \\
\text { the worse } \\
\text { outcome with } \\
\text { higher levels of } \\
\text { proteinuria and } \\
\text { blood pressure } \\
\text { than white and } \\
\text { South Asia group. } \\
\text { Severe CKD is } \\
\text { higher among } \\
\text { black and South } \\
\text { Asian populations } \\
\text { with mild CKD } \\
\text { higher among } \\
\text { white population. }\end{array}$ \\
\hline
\end{tabular}




\begin{tabular}{|c|c|c|c|c|c|c|}
\hline Study & Aim & Study design & $\begin{array}{l}\text { Study } \\
\text { quality }\end{array}$ & $\begin{array}{l}\text { Participant's } \\
\text { demography }\end{array}$ & Key findings & Conclusion \\
\hline & & & & & $\begin{array}{l}\text { 130/80mmHg. } \\
\text { Black population } \\
\text { receiving } \\
\text { prescription } \\
\text { more than South } \\
\text { Asia or white } \\
\text { groups. }\end{array}$ & \\
\hline $\begin{array}{l}\text { James et } \\
\text { al. } 2012^{36}\end{array}$ & $\begin{array}{l}\text { To describe the } \\
\text { independent } \\
\text { influence of } \\
\text { both ethnic and } \\
\text { social group on } \\
\text { HbA1C levels } \\
\text { in people with } \\
\text { type } 2 \text { diabetes } \\
\text { routinely cared } \\
\text { for by general } \\
\text { practice for } \\
\text { over } 5 \text { years. }\end{array}$ & $\begin{array}{l}\text { Quantitative } \\
\text { Cross- } \\
\text { sectional } \\
\text { from Web- } \\
\text { enabled } \\
\text { computer } \\
\text { System }\end{array}$ & $\begin{array}{l}18 / 20 \\
90 \%\end{array}$ & $\begin{array}{l}\text { Whites: 5,206 } \\
\text { (22\%), Black } \\
\text { Africa/ } \\
\text { Caribbean: } \\
\text { 3,923 (17\%), } \\
\text { South Asia: } \\
\text { 13,633 (58\%) } \\
\text { and Others } \\
721 \text { (3\%). Age } \\
\text { Range: } 35-75 \\
\text { years. Sample } \\
\text { size: } 24,111 .\end{array}$ & $\begin{array}{l}\text { White (69\%) } \\
\text { people were less } \\
\text { likely to be on } \\
\text { intensive } \\
\text { diabetes } \\
\text { treatment } \\
\text { (Combined oral } \\
\text { or Insulin) than } \\
\text { South Asia (75\%) } \\
\text { and black } \\
\text { African/ } \\
\text { Caribbean } \\
\text { population } \\
\text { (73\%). Mean of } \\
\text { HbA1C declined } \\
\text { in white group } \\
\text { by 0.4\% from } \\
8.2 \% \text { to } 7.8 \% \text { : } \\
0.5 \% \text { for South } \\
\text { Asian population } \\
\text { and Africa/ } \\
\text { Caribbean group } \\
\text { from } 8.5 \% \text { to } \\
8.0 \% \text {. The } \\
\text { proportion of } \\
\text { people with } \\
7.5 \% \text { or less } \\
\text { HbA1C } \\
\text { increased by } \\
12 \% \text { in White } \\
\text { group, 14\% in } \\
\text { South Asia and } \\
15 \% \text { in Africa/ } \\
\text { Caribbean } \\
\text { groups. }\end{array}$ & $\begin{array}{l}\text { There was } \\
\text { improvement in } \\
\text { HBA1C among all } \\
\text { ethnic groups. } \\
\text { However, ethnic } \\
\text { differences still } \\
\text { persisted. Ethnic } \\
\text { group and social } \\
\text { deprivation are } \\
\text { independently } \\
\text { associated with } \\
\text { HbA1C. }\end{array}$ \\
\hline
\end{tabular}

\begin{tabular}{|c|c|c|c|c|c|c|}
\hline $\begin{array}{l}\text { Kahn et } \\
\text { al. } 2012^{37}\end{array}$ & $\begin{array}{l}\text { To examine } \\
\text { multi-ethnic } \\
\text { participants' } \\
\text { explanation of } \\
\text { how their } \\
\text { diabetes } \\
\text { began, } \\
\text { understandings } \\
\text { about their } \\
\text { illness, } \\
\text { description of } \\
\text { the symptoms } \\
\text { experience. To } \\
\text { analyse the } \\
\text { extent to which } \\
\text { themes } \\
\text { persisted } \\
\text { across ethnic, } \\
\text { cultural and } \\
\text { racial } \\
\text { boundaries }\end{array}$ & $\begin{array}{l}\text { Qualitative } \\
\text { approach } \\
\text { using semi- } \\
\text { structured } \\
\text { interviews }\end{array}$ & $\begin{array}{l}17 / 20 \\
85 \%\end{array}$ & $\begin{array}{l}\text { Refugee } \\
\text { (Somalia, } \\
\text { Sudan, Burma } \\
\text { or Cuba). } \\
\text { Sample Size: } \\
\text { 34. Male: } 8 \text {, } \\
\text { Female: } 26 . \\
\text { Education: } 13 \\
\text { secondary } \\
\text { school } \\
\text { completion }\end{array}$ & $\begin{array}{l}\text { Unexpected and } \\
\text { late diagnosis of } \\
\text { diabetes was } \\
\text { reported. } \\
\text { Reaction to } \\
\text { living with } \\
\text { diagnosis was } \\
\text { reported as } \\
\text { grief, anger, } \\
\text { depressive } \\
\text { symptoms, and } \\
\text { acceptance. } \\
\text { Most patients } \\
\text { understanding is } \\
\text { focused on } \\
\text { symptoms and } \\
\text { diet }\end{array}$ & $\begin{array}{l}\text { People living with } \\
\text { diabetes express } \\
\text { emotions similar } \\
\text { to dying patients. } \\
\text { There is a need for } \\
\text { practitioners to } \\
\text { include patients as } \\
\text { partners in the } \\
\text { development of } \\
\text { patient centred } \\
\text { approach to } \\
\text { diabetes } \\
\text { management }\end{array}$ \\
\hline $\begin{array}{l}\text { Kindarara } \\
\text { et al. } \\
2017^{38}\end{array}$ & $\begin{array}{l}\text { To describe } \\
\text { Sub-Saharan } \\
\text { African } \\
\text { immigrants' } \\
\text { health-illness }\end{array}$ & $\begin{array}{l}\text { Qualitative } \\
\text { approach } \\
\text { using face-to- } \\
\text { face semi- } \\
\text { structured }\end{array}$ & $\begin{array}{l}18 / 20 \\
90 \%\end{array}$ & $\begin{array}{l}\text { Sub-Sharan } \\
\text { African } \\
\text { immigrants } \\
\text { Sample Size: } \\
\text { 10. Mean Age: }\end{array}$ & $\begin{array}{l}\text { Participants } \\
\text { reported limited } \\
\text { knowledge } \\
\text { about diabetes. } \\
\text { Dealing with the }\end{array}$ & $\begin{array}{l}\text { Professionals } \\
\text { need to access } \\
\text { and recognise } \\
\text { inhibitors that can } \\
\text { influence diabetes }\end{array}$ \\
\hline
\end{tabular}




\begin{tabular}{|c|c|c|c|c|c|c|}
\hline Study & Aim & Study design & $\begin{array}{l}\text { Study } \\
\text { quality }\end{array}$ & $\begin{array}{l}\text { Participant's } \\
\text { demography }\end{array}$ & Key findings & Conclusion \\
\hline & $\begin{array}{l}\text { transition } \\
\text { experiences } \\
\text { associated with } \\
\text { type } 2 \text { diabetes } \\
\text { mellitus self- } \\
\text { management }\end{array}$ & $\begin{array}{l}\text { in-depth } \\
\text { interview }\end{array}$ & & $\begin{array}{l}60.3 \text { years. } \\
\text { Sampling: } \\
\text { Purposive and } \\
\text { Snowballing } \\
\text { Sampling. } \\
\text { Male: } 5 \text {, } \\
\text { Female: } 5\end{array}$ & $\begin{array}{l}\text { shock of } \\
\text { diagnosis with } \\
\text { diabetes, } \\
\text { cultural beliefs } \\
\text { can be inhibitors } \\
\text { of self- } \\
\text { management }\end{array}$ & self-management. \\
\hline $\begin{array}{l}\text { Kohinor } \\
\text { et al. } \\
2011^{39}\end{array}$ & $\begin{array}{l}\text { To determine } \\
\text { the social- } \\
\text { cultural factors } \\
\text { affecting the } \\
\text { dietary } \\
\text { behaviour of } \\
\text { Dutch } \\
\text { Surinamese } \\
\text { patients with } \\
\text { type } 2 \text { diabetes }\end{array}$ & $\begin{array}{l}\text { Qualitative } \\
\text { approach } \\
\text { using } \\
\text { Grounded } \\
\text { theory } \\
\text { methodology }\end{array}$ & $\begin{array}{l}18 / 20 \\
90 \%\end{array}$ & $\begin{array}{l}\text { African } \\
\text { Surinamese } \\
\text { Hindustani } \\
\text { Surinamese. } \\
\text { Sample Size: } \\
\text { 32. Mean Age: } \\
\text { 55. Male: } 12 \\
\text { Female: } 20\end{array}$ & $\begin{array}{l}\text { Participants } \\
\text { reported finding } \\
\text { it difficult to } \\
\text { choose good } \\
\text { food products, } \\
\text { Holding on to } \\
\text { their traditional } \\
\text { food as identity, } \\
\text { culture plays } \\
\text { important role in } \\
\text { their food } \\
\text { preparation. }\end{array}$ & $\begin{array}{l}\text { Immigrants } \\
\text { continue with } \\
\text { their country of } \\
\text { origin food and } \\
\text { cultural } \\
\text { considerations } \\
\text { should be involved } \\
\text { in advising people } \\
\text { with diabetes on } \\
\text { dietary } \\
\text { recommendations }\end{array}$ \\
\hline $\begin{array}{l}\text { Snider et } \\
\text { al. } 2017^{40}\end{array}$ & $\begin{array}{l}\text { To explore both } \\
\text { the age- } \\
\text { specific } \\
\text { prevalence of } \\
\text { diabetes and } \\
\text { the current } \\
\text { level of } \\
\text { awareness, } \\
\text { medical } \\
\text { treatment and } \\
\text { glycaemic } \\
\text { control among } \\
\text { different } \\
\text { ethnic groups. }\end{array}$ & $\begin{array}{l}\text { Quantitative } \\
\text { cross- } \\
\text { sectional } \\
\text { study }\end{array}$ & $\begin{array}{l}\text { 18/20, } \\
90 \%\end{array}$ & $\begin{array}{l}\text { Dutch 4,541, } \\
\text { South Asia } \\
\text { Surinamese } \\
\text { 3,032. African } \\
\text { Surinamese } \\
\text { 4,109, } \\
\text { Ghanaian } \\
\text { 2,232, Turkish } \\
\text { 3,591 and } \\
\text { Moroccan } \\
\text { 3,887. Sample } \\
\text { size: } 21,483 . \\
\text { Sampling: } \\
\text { Municipal } \\
\text { register. Age } \\
\text { range: } 18-70\end{array}$ & $\begin{array}{l}\text { Diabetes } \\
\text { prevalence } \\
\text { increased among } \\
\text { ethnic groups } \\
\text { with age. Higher } \\
\text { than Dutch and } \\
\text { significant from } \\
\text { age } 31-40 \text { years. } \\
\text { There was } \\
\text { higher } \\
\text { awareness of } \\
\text { diabetes } \\
\text { (70-80\%) among } \\
\text { ethnic groups } \\
\text { compared to } \\
\text { Dutch (60\%). } \\
\text { The odd for } \\
\text { receiving } \\
\text { medical } \\
\text { treatment for } \\
\text { diabetes is also } \\
\text { higher among all } \\
\text { ethnic minority } \\
\text { compared to } \\
\text { Dutch. All ethnic } \\
\text { minority men } \\
\text { are significantly } \\
\text { lower odds of } \\
\text { controlled } \\
\text { HbA1C than } \\
\text { Dutch }\end{array}$ & $\begin{array}{l}\text { Ethnic groups } \\
\text { have higher } \\
\text { prevalence of } \\
\text { diabetes, although } \\
\text { awareness is a } \\
\text { higher than } \\
\text { among Dutch. } \\
\text { There was } \\
\text { significant lower } \\
\text { control of HbA1C } \\
\text { among ethnic } \\
\text { minority men than } \\
\text { Dutch but no } \\
\text { difference among } \\
\text { women. There is } \\
\text { need to } \\
\text { understand the } \\
\text { cause of poor } \\
\text { glycaemic control } \\
\text { among ethnic } \\
\text { minority. }\end{array}$ \\
\hline $\begin{array}{l}\text { Verma et } \\
\text { al. } 2010^{41}\end{array}$ & $\begin{array}{l}\text { To determine } \\
\text { the impact of } \\
\text { quality } \\
\text { improvement } \\
\text { initiatives on } \\
\text { ethnic } \\
\text { disparities in } \\
\text { diabetes } \\
\text { management in } \\
\text { the UK }\end{array}$ & $\begin{array}{l}\text { Quantitative } \\
\text { cross- } \\
\text { sectional } \\
\text { survey }\end{array}$ & $\begin{array}{l}\text { 18/20, } \\
90 \%\end{array}$ & $\begin{array}{l}4309 \\
\text { Participants. } \\
\text { white } \\
\text { population: } \\
\text { 13.7\%, black } \\
\text { population: } \\
\text { 16.1\%. South } \\
\text { Asians: } 51.2 \% \text {, } \\
\text { Others: } 18.3 \% \text {. } \\
\text { Age: } 18 \text { and } \\
\text { above. Male: } \\
\text { 2393 (55.5\%) } \\
\text { Female: } 1871 \\
\text { (43.4\%). No } \\
\text { sex: } 45 \text { (1.0\%) }\end{array}$ & $\begin{array}{l}\text { No difference in } \\
\text { evidence of the } \\
\text { process of care } \\
\text { among all ethnic } \\
\text { groups The } \\
\text { Proportion of } \\
\text { patients meeting } \\
\text { national } \\
\text { treatment } \\
\text { targets for Bp, } \\
\text { cholesterol and } \\
\text { HbA1C } \\
\text { increased from } \\
\text { 1997-2007. } \\
\text { Black patients } \\
\text { achieving the } \\
\text { targets doubled } \\
\text { but still remain }\end{array}$ & $\begin{array}{l}\text { There has been } \\
\text { improvement in } \\
\text { patients meeting } \\
\text { the three targets } \\
\text { since } 1997 . \\
\text { However, less } \\
\text { than } 20 \% \text { were } \\
\text { able to meet this } \\
\text { target. Medication } \\
\text { prescription also } \\
\text { increased for all } \\
\text { ethnic groups. } \\
\text { There is need for } \\
\text { better } \\
\text { improvement in } \\
\text { care and } \\
\text { management. }\end{array}$ \\
\hline
\end{tabular}




\begin{tabular}{|c|c|c|c|c|c|c|}
\hline Study & Aim & Study design & $\begin{array}{l}\text { Study } \\
\text { quality }\end{array}$ & $\begin{array}{l}\text { Participant's } \\
\text { demography }\end{array}$ & Key findings & Conclusion \\
\hline & & & & & $\begin{array}{l}\text { less likely to } \\
\text { meet target in } \\
2006 \text { compared } \\
\text { to white group. } \\
\text { Black population } \\
\text { were less likely } \\
\text { to meet all three } \\
\text { targets than } \\
\text { white group. } \\
\text { South Asians } \\
\text { were more likely } \\
\text { to meet } \\
\text { cholesterol } \\
\text { target than } \\
\text { white population } \\
\text { by } 2006 \text {. There } \\
\text { is increase } \\
\text { prescription of } \\
\text { lipid-lowering, } \\
\text { oral } \\
\text { hypoglycaemic } \\
\text { agents, insulin } \\
\text { and } \\
\text { antihypertensive } \\
\text { medications } \\
\text { since } 1997 . \\
\text { Increase in } \\
\text { prescription } \\
\text { medication for } \\
\text { black population } \\
\text { but lower lipid- } \\
\text { lowering } \\
\text { medication than } \\
\text { white patients in } \\
2006 \text {. Black } \\
\text { patients were } \\
\text { more likely to be } \\
\text { on oral } \\
\text { hypoglycaemia } \\
\text { agent than white } \\
\text { population. }\end{array}$ & \\
\hline $\begin{array}{l}\text { Fosse- } \\
\text { Edorh et } \\
\text { al. } 2014^{42}\end{array}$ & $\begin{array}{l}\text { To present an } \\
\text { overview of } \\
\text { type } 2 \text { diabetes } \\
\text { among North } \\
\text { African } \\
\text { immigrants in } \\
\text { France }\end{array}$ & $\begin{array}{l}\text { Quantitative } \\
\text { cross- } \\
\text { sectional } \\
\text { study using } \\
\text { national } \\
\text { survey }\end{array}$ & $\begin{array}{l}17 / 20 \\
85 \%\end{array}$ & $\begin{array}{l}\text { Race: Africans. } \\
\text { France Sample } \\
\text { technique: } \\
\text { National } \\
\text { survey } \\
\text { records. } \\
\text { Sample size: } \\
\text { Born in North } \\
\text { Africa (BNA). } \\
\text { Male: 191. } \\
\text { Born in France } \\
\text { (BIF) Male: } \\
\text { 5821 BNA } \\
\text { (Female) 136. } \\
\text { BIF (Female) } \\
6890 . \text { Mean } \\
\text { Age: BNA } \\
\text { (Male) 58. BIF } \\
\text { (Male) 61. } \\
\text { BNA (Female) } \\
56 \text { BIF } \\
\text { (Female) 63. } \\
\text { Context: } \\
\text { France. } \\
\text { Ethnicity: } \\
\text { black and } \\
\text { white } \\
\text { populations. }\end{array}$ & $\begin{array}{l}\text { Type } 2 \text { diabetes } \\
\text { prevalence and } \\
\text { obesity is higher } \\
\text { among BNA } \\
\text { than BIF. HbA1c } \\
\text { is also higher } \\
\text { among BNA } \\
\text { than BIF which } \\
\text { indicate poorer } \\
\text { control among } \\
\text { this population. }\end{array}$ & $\begin{array}{l}\text { Although a higher } \\
\text { prevalence of type } \\
2 \text { diabetes and } \\
\text { poorer glycaemic } \\
\text { control was } \\
\text { reported among } \\
\text { BNA women. } \\
\text { There is a poorer } \\
\text { control among } \\
\text { both Male and } \\
\text { Female BNA than } \\
\text { BIF contributing } \\
\text { to complication } \\
\text { disparity among } \\
\text { this population }\end{array}$ \\
\hline
\end{tabular}




\begin{tabular}{|c|c|c|c|c|c|c|}
\hline Study & Aim & Study design & $\begin{array}{l}\text { Study } \\
\text { quality }\end{array}$ & $\begin{array}{l}\text { Participant's } \\
\text { demography }\end{array}$ & Key findings & Conclusion \\
\hline $\begin{array}{l}\text { Wieland } \\
\text { et al. } \\
2012^{43}\end{array}$ & $\begin{array}{l}\text { To measure } \\
\text { outcomes of } \\
\text { diabetes care } \\
\text { among Somali } \\
\text { immigrants }\end{array}$ & $\begin{array}{l}\text { Quantitative } \\
\text { methodology }\end{array}$ & $\begin{array}{l}16 / 20 \\
80 \%\end{array}$ & $\begin{array}{l}\text { Race: Somalia } \\
\text { And Non- } \\
\text { Somalia. } \\
\text { Sample } \\
\text { technique: } \\
\text { Medical } \\
\text { record. Sample } \\
\text { size: } 81 . \\
\text { Somalian } \\
\text { 5,843, Non- } \\
\text { Somalian. } \\
\text { Mean Age: not } \\
\text { mentioned. } \\
\text { Context: USA. } \\
\text { Socioeconomic } \\
\text { status: Not } \\
\text { mentioned. } \\
\text { Ethnicity: } \\
\text { black } \\
\text { population and } \\
\text { Others }\end{array}$ & $\begin{array}{l}\text { Somalians were } \\
\text { less likely to } \\
\text { meet the } \\
\text { optimum HbA1C } \\
\text { control < } 7 \% \text {. } \\
\text { There was no } \\
\text { significant } \\
\text { difference in the } \\
\text { lipid control } \\
\text { level among the } \\
\text { Somali and Non- } \\
\text { Somali groups. } \\
\text { Also, there was } \\
\text { no difference in } \\
\text { the achievement } \\
\text { of blood } \\
\text { pressure control } \\
\text { between the two } \\
\text { groups. }\end{array}$ & $\begin{array}{l}\text { There is a } \\
\text { disparity in } \\
\text { diabetes control } \\
\text { among Somali } \\
\text { immigrants living } \\
\text { with diabetes as } \\
\text { compared to Non- } \\
\text { Somali groups. } \\
\text { This might be due } \\
\text { to medical } \\
\text { preference, } \\
\text { socioeconomic } \\
\text { factors, health } \\
\text { literacy and } \\
\text { culture. } \\
\text { Community- } \\
\text { practice based } \\
\text { intervention is } \\
\text { needed to } \\
\text { improve diabetes } \\
\text { management } \\
\text { among this } \\
\text { vulnerable } \\
\text { population group. }\end{array}$ \\
\hline $\begin{array}{l}\text { Wallin et } \\
\text { al. } 2007^{44}\end{array}$ & $\begin{array}{l}\text { To explore the } \\
\text { daily life } \\
\text { experience of } \\
\text { Somalian } \\
\text { diabetic } \\
\text { patients living } \\
\text { in Sweden with } \\
\text { gender-related } \\
\text { perspectives to } \\
\text { diabetes- } \\
\text { related } \\
\text { problems } \\
\text { management }\end{array}$ & $\begin{array}{l}\text { Qualitative } \\
\text { methodology } \\
\text { Interviews }\end{array}$ & $\begin{array}{l}16 / 20 \\
80 \%\end{array}$ & $\begin{array}{l}\text { Race: } \\
\text { Somalian } \\
\text { Sample } \\
\text { Technique: } \\
\text { Not } \\
\text { mentioned } \\
\text { Sample size: } \\
19 \\
\text { Participants } \\
\text { interviewed } \\
\text { with } \\
\text { interpreter's } \\
\text { help. Mean } \\
\text { Age: } 54.9 \\
\text { Context: } \\
\text { Sweden } \\
\text { Socioeconomic } \\
\text { status: Not } \\
\text { mentioned } \\
\text { Ethnicity: } \\
\text { black group }\end{array}$ & $\begin{array}{l}\text { Experience of } \\
\text { distress in daily } \\
\text { life as } \\
\text { participants find } \\
\text { it difficult to } \\
\text { maintain daily } \\
\text { activities. } \\
\text { Difficulty to } \\
\text { follow the } \\
\text { dietary advice } \\
\text { by health } \\
\text { professional }\end{array}$ & $\begin{array}{l}\text { Cultural } \\
\text { consideration is } \\
\text { essential in health } \\
\text { promotional } \\
\text { services for } \\
\text { immigrants. } \\
\text { Religion and } \\
\text { gender } \\
\text { consideration is } \\
\text { also essential in } \\
\text { the prevention } \\
\text { and management } \\
\text { of diabetes among } \\
\text { this ethnic group. }\end{array}$ \\
\hline $\begin{array}{l}\text { Choukem } \\
\text { et al. } \\
2014^{45}\end{array}$ & $\begin{array}{l}\text { To determine } \\
\text { the } \\
\text { contribution of } \\
\text { migration on } \\
\text { the } \\
\text { characteristic } \\
\text { of Type } 2 \\
\text { diabetes } \\
\text { comparing } \\
\text { three } \\
\text { populations } \\
\text { living with } \\
\text { diabetes }\end{array}$ & $\begin{array}{l}\text { Quantitative } \\
\text { cross- } \\
\text { sectional } \\
\text { study design }\end{array}$ & $\begin{array}{l}19 / 20 \\
95 \%\end{array}$ & $\begin{array}{l}\text { Race: } \\
\text { Cameroonians, } \\
\text { Caucasians. } \\
\text { Sample } \\
\text { technique: } \\
\text { Cross- } \\
\text { sectional } \\
\text { survey. Sample } \\
\text { size: } \\
\text { Cameroonian } \\
\text { 100, African } \\
\text { immigrants 98, } \\
\text { Caucasians } \\
\text { 199. Age } \\
\text { Range: } \\
\text { Cameroonian } \\
\text { 30-80, African } \\
\text { migrant 26-75, } \\
\text { Caucasian } \\
\text { 28-89. } \\
\text { Context: } \\
\text { France. } \\
\text { Socioeconomic } \\
\text { status: Not }\end{array}$ & $\begin{array}{l}\text { Diabetes was } \\
\text { diagnosed at a } \\
\text { later age among } \\
\text { Cameroonian. } \\
\text { There were no } \\
\text { differences } \\
\text { among } \\
\text { Cameroonians } \\
\text { and African } \\
\text { immigrants in } \\
\text { mean BMI, } \\
\text { overweight, } \\
\text { obesity and } \\
\text { smoking but } \\
\text { higher among } \\
\text { Caucasians. } \\
\text { Cameroonians } \\
\text { had the highest } \\
\text { rate of } \\
\text { microvascular } \\
\text { complications } \\
\text { than the other } \\
\text { groups. }\end{array}$ & $\begin{array}{l}\text { Cameroonians are } \\
\text { diagnosed with } \\
\text { diabetes at a later } \\
\text { age but present } \\
\text { with higher } \\
\text { complications } \\
\text { than African } \\
\text { immigrants and } \\
\text { Caucasians which } \\
\text { might be due to } \\
\text { delayed diagnosis } \\
\text { and poorer } \\
\text { management } \\
\text { among the } \\
\text { Cameroonian } \\
\text { population. }\end{array}$ \\
\hline
\end{tabular}




\begin{tabular}{|c|c|c|c|c|c|c|}
\hline Study & Aim & Study design & $\begin{array}{l}\text { Study } \\
\text { quality }\end{array}$ & $\begin{array}{l}\text { Participant's } \\
\text { demography }\end{array}$ & Key findings & Conclusion \\
\hline & & & & $\begin{array}{l}\text { mentioned. } \\
\text { Ethnicity: } \\
\text { black and } \\
\text { white } \\
\text { population }\end{array}$ & & \\
\hline $\begin{array}{l}\text { Abubakari } \\
\text { et al. } \\
2013^{46}\end{array}$ & $\begin{array}{l}\text { To investigate } \\
\text { diabetes } \\
\text { knowledge and } \\
\text { illness } \\
\text { perception on } \\
\text { self- } \\
\text { management } \\
\text { and also to } \\
\text { determine the } \\
\text { relationship } \\
\text { between self- } \\
\text { management } \\
\text { behaviour and } \\
\text { glycaemic } \\
\text { control among } \\
\text { African-Origin } \\
\text { patients in the } \\
\text { UK. }\end{array}$ & $\begin{array}{l}\text { Quantitative } \\
\text { Methodology }\end{array}$ & $\begin{array}{l}18 / 20 \\
90 \%\end{array}$ & $\begin{array}{l}\text { Race: White } \\
\text { British, African } \\
\text { Caribbean and } \\
\text { black Africans. } \\
\text { Sample } \\
\text { technique: } \\
\text { Convenience } \\
\text { sampling. } \\
\text { Sample size: } \\
137 \text { white } \\
\text { British, } 123 \\
\text { African } \\
\text { Caribbean and } \\
\text { 99 black } \\
\text { Africans. } \\
\text { Context: UK. } \\
\text { Socioeconomic } \\
\text { status: Not } \\
\text { mentioned. } \\
\text { Ethnicity: } \\
\text { white and } \\
\text { black } \\
\text { population. }\end{array}$ & $\begin{array}{l}\text { High knowledge } \\
\text { about diabetes } \\
\text { does not } \\
\text { influence better } \\
\text { self- } \\
\text { management in } \\
\text { white group and } \\
\text { was related to } \\
\text { less self- } \\
\text { management in } \\
\text { Africans. High } \\
\text { Illness } \\
\text { perception } \\
\text { among white- } \\
\text { British was } \\
\text { associated with } \\
\text { less exercise } \\
\text { self- } \\
\text { management. In } \\
\text { Africans, high } \\
\text { illness } \\
\text { perception was } \\
\text { associated with } \\
\text { fewer feet } \\
\text { management } \\
\text { and dietary } \\
\text { regulation. } \\
\text { Perceived } \\
\text { personal control } \\
\text { was related to } \\
\text { frequent overall } \\
\text { self- } \\
\text { management }\end{array}$ & $\begin{array}{l}\text { Type } 2 \text { diabetes } \\
\text { knowledge and } \\
\text { perception varies } \\
\text { between ethnic } \\
\text { groups in the UK } \\
\text { which might } \\
\text { influence the } \\
\text { disease } \\
\text { management } \\
\text { outcome. These } \\
\text { perceptions need } \\
\text { to be identified } \\
\text { and any } \\
\text { misconceptions } \\
\text { corrected to allow } \\
\text { for efficient self- } \\
\text { management } \\
\text { recommendations } \\
\text { for this } \\
\text { population. }\end{array}$ \\
\hline $\begin{array}{l}\text { Bijlholt et } \\
\text { al. } 2018^{47}\end{array}$ & $\begin{array}{l}\text { To asses } \\
\text { differences in } \\
\text { awareness, } \\
\text { treatment and } \\
\text { control of } \\
\text { diabetes } \\
\text { among a } \\
\text { relatively } \\
\text { homogeneous } \\
\text { population } \\
\text { from } \\
\text { Ghanaians } \\
\text { living in } \backslash \text { Rural, } \\
\text { urban parts of } \\
\text { Ghana and } \\
\text { Ghanaian } \\
\text { immigrants } \\
\text { living in } \\
\text { European cities }\end{array}$ & $\begin{array}{l}\text { Quantitative } \\
\text { Cross- } \\
\text { sectional } \\
\text { study }\end{array}$ & $\begin{array}{l}19 / 20 \\
95 \%\end{array}$ & $\begin{array}{l}\text { Race: Black } \\
\text { population. } \\
\text { Sample } \\
\text { selection: } \\
\text { Purposive. } \\
\text { Sample size: } \\
\text { 530. } \\
\text { Amsterdam } \\
\text { 172, Berlin 70, } \\
\text { London 102, } \\
\text { Urban Ghana } \\
\text { 135, Rural } \\
\text { Ghana 51. } \\
\text { Mean Age: } \\
\text { Amsterdam } \\
\text { 52.2, Berlin } \\
\text { 51.1, London } \\
\text { 54.6, Urban } \\
\text { Ghana 52.9, } \\
\text { Rural Ghana } \\
\text { 54.5. Gender: } \\
\text { Male and } \\
\text { Female. } \\
\text { Context: } \\
\text { Amsterdam, } \\
\text { Berlin, London } \\
\text { Ghana, } \\
\text { Ethnicity: } \\
\text { black-Africans } \\
\text { (100\%) }\end{array}$ & $\begin{array}{l}\text { Type } 2 \text { diabetes } \\
\text { awareness was } \\
\text { lowest among } \\
\text { people in rural } \\
\text { Ghana and } \\
\text { highest in } \\
\text { European sites } \\
\text { (Amsterdam, } \\
\text { berlin and } \\
\text { London). } \\
\text { Diabetes control } \\
\text { was similar in } \\
\text { Amsterdam, } \\
\text { Berlin and rural } \\
\text { Ghana but lower } \\
\text { in urban Ghana } \\
\text { and lowest in } \\
\text { London. }\end{array}$ & $\begin{array}{l}\text { Although type } 2 \\
\text { diabetes } \\
\text { awareness and } \\
\text { treatment rates } \\
\text { were lowest in } \\
\text { rural Ghana, type } \\
2 \text { diabetes control } \\
\text { was lowest in } \\
\text { London and urban } \\
\text { Ghana sites. }\end{array}$ \\
\hline
\end{tabular}




\begin{tabular}{|c|c|c|c|c|c|c|}
\hline Study & Aim & Study design & $\begin{array}{l}\text { Study } \\
\text { quality }\end{array}$ & $\begin{array}{l}\text { Participant's } \\
\text { demography }\end{array}$ & Key findings & Conclusion \\
\hline $\begin{array}{l}\text { Brämberg } \\
\text { et al } \\
2012^{48}\end{array}$ & $\begin{array}{l}\text { To describe the } \\
\text { care provided } \\
\text { by a Diabetes } \\
\text { Nurse } \\
\text { Specialist } \\
\text { (DNS) and the } \\
\text { care needs } \\
\text { expressed by } \\
\text { immigrants } \\
\text { living with type } \\
2 \text { diabetes }\end{array}$ & $\begin{array}{l}\text { Qualitative } \\
\text { observational } \\
\text { study }\end{array}$ & $\begin{array}{l}16 / 20 \\
80 \%\end{array}$ & $\begin{array}{l}\text { Race: black } \\
\text { Caribbean } \\
\text { (BC) and } \\
\text { Middle-East. } \\
\text { Sample } \\
\text { selection: } \\
\text { Purposive. } \\
\text { Sample size: } \\
10 \\
\text { observation of } \\
\text { consultation } \\
\text { interview. } \\
\text { Gender: Male } \\
\text { and Female. } \\
\text { Context: } \\
\text { Sweden }\end{array}$ & $\begin{array}{l}\text { There was } \\
\text { power } \\
\text { imbalance with } \\
\text { patients passive } \\
\text { during the } \\
\text { consultation. } \\
\text { There was } \\
\text { limited support } \\
\text { provided by } \\
\text { DNS in } \\
\text { addressing } \\
\text { patient's } \\
\text { concerns due to } \\
\text { lack of } \\
\text { individualised } \\
\text { care. There was } \\
\text { limited support } \\
\text { provided by } \\
\text { DNS in } \\
\text { addressing } \\
\text { patient's } \\
\text { concerns due to } \\
\text { lack of } \\
\text { individualised } \\
\text { care }\end{array}$ & $\begin{array}{l}\text { Balanced } \\
\text { communication is } \\
\text { urgently needed. } \\
\text { Person-centred } \\
\text { consultation and } \\
\text { care for people } \\
\text { from immigrant } \\
\text { background is } \\
\text { seen as an } \\
\text { important } \\
\text { approach to } \\
\text { diabetes } \\
\text { management } \\
\text { among this } \\
\text { population. }\end{array}$ \\
\hline
\end{tabular}

$\mathrm{S} / \mathrm{N}$ - Serial Number

Table 3. Biomedical target outcomes based on different health organisations

\begin{tabular}{lll}
\hline Organisation & Target & Citations \\
\hline HbA1C & $<7 \%(53 \mathrm{mmol} / \mathrm{L})$ & IDF $2017^{51}$ \\
IDF & $<6.5 \%(48 \mathrm{mmol} / \mathrm{L})$ & WHO $2007^{52}$ \\
WHO & $<6.5 \%(48 \mathrm{mmol} / \mathrm{L})$ & NICE $2015^{53}$ \\
$\mathrm{NICE}$ & $<7 \%(53 \mathrm{mmol} / \mathrm{L})$ & ADA $2016^{54}$ \\
ADA & & \\
Blood Pressure & Systolic: $130-140 \mathrm{mmHg}$ & \\
IDF & Diastolic: $80 \mathrm{mmHg}$ & IDF $2017^{51}$ \\
WHO & $130 / 80 \mathrm{mmHg}$ & WHO $2007^{52}$ \\
NICE & $<140 / 80 \mathrm{mmHg}$ & NICE $2009^{55}$ \\
ADA & $<140 / 90 \mathrm{mmHg}$ & De Boer et al. $2017^{56}$ \\
Cholesterol & & \\
IDF & LDL cholesterol $<2.6 \mathrm{mmol} / \mathrm{L}$ & IDF $2017^{51}$ \\
WHO & Total cholesterol $<4.0 \mathrm{mmol} / \mathrm{L}(152 \mathrm{mg} / \mathrm{dll})$ & WHO $2007^{52}$ \\
& LDL cholesterol $<2.0 \mathrm{mmol} / \mathrm{L}(77 \mathrm{mg} / \mathrm{dl})$ & \\
NICE & LDL cholesterol $<2.0 \mathrm{mmol} / \mathrm{L}$ & NICE $2012^{57}$ \\
ADA & HDL cholesterol $<1.4 \mathrm{mmol} / \mathrm{L}$ & \\
\hline
\end{tabular}

IDF - International Diabetes Federation, WHO - World Health Organization, NICE - National Institute for Health and Care Excellence, ADA - American Diabetes Association

the black population. black population are defined as the groups of individuals with dark-skin and used for racial classification. ${ }^{61}$ black African which is a specific group in black population is used to describe individuals that originate from Sub-Saharan African countries. African Caribbean is used to refer to individuals that originated from Caribbean countries such as Jamaica, Trinidad and Tobago. Finally, African American refers to individuals that are citizen of America. Although the ancestral linage of African Caribbean and African American originates from Sub-Saharan African countries, the influence of slave trade has caused their movement to continents outside Africa. 
Table 5. Diabetes prevalence reported in reviewed studies

\begin{tabular}{|c|c|c|c|}
\hline Diabetes prevalence (\%) & & & \\
\hline Authors & Black Africans & South Asians & White group \\
\hline \multirow{5}{*}{ Ballotari et al. $2015^{34}$} & Male: 4.3 & Male: 10.2 & Male: 5.5 \\
\hline & Female: 6.5 & Female: 9.7 & Female: 3.6 \\
\hline & Black-Caribbean & & \\
\hline & Male: 3.2 & & \\
\hline & Female: 4.3 & & \\
\hline James et al. $2012^{36}$ & \multicolumn{3}{|c|}{ NR } \\
\hline Dreyer et al. $2009^{35}$ & 8 & 11 & 3.5 \\
\hline \multirow{2}{*}{ Snijder et al. $2017^{40}$} & Male: 14.9 & Male: 21.5 & Male: 5.0 \\
\hline & Female: 9.6 & Female: 12.2 & Female: 2.4 \\
\hline \multirow{2}{*}{ Fosse-Edorh et al. $2014^{42}$} & Male: 12 & NR & Male: 8.6 \\
\hline & Female: 17 & Female: 6.5 & \\
\hline Weiland et al. $2012^{43}$ & & NR & \\
\hline Bijlholt et al. $2018^{47}$ & & NR & \\
\hline Choukem et al. $2014^{45}$ & & NR & \\
\hline Abubakari et al. $2013^{46}$ & & NR & \\
\hline Verma et al. $2010^{41}$ & & NR & \\
\hline
\end{tabular}

NR - not reported

Using phenotypic, racial classification for these groups will result in classification as one group. However, classifying using ethnicity will result in at least the three major groups described above. This is mainly because classifying using ethnicity goes beyond phenotypic characteristics to include characteristics such as language, diet, religion, geography. ${ }^{61}$ The importance of specific classifications in this review is due to the aim of the review. It is therefore essential to differentiate the black African immigrant population as the population of interest from other subset groups of black population. ${ }^{62}$ The interest in black Africans is because this group is the smaller than African Caribbean and African-American and is underrepresented in healthcare research.

In the search of studies for this review, it was noted that majority of the articles retrieved recruited African Americans in the USA and African Caribbean in the UK. To meet the aim of this review, studies that only recruit African American or African Caribbean were excluded. Studies have to recruit black African immigrants to be included in this review for analysis.

\section{DIABETES PREVALENCE}

Diabetes prevalence was one of the results reported among studies reviewed. This was reported by four studies included in this review. ${ }^{34,35,40,42}$ Diabetes prevalence was reported to be higher among black Africans and South Asians than the white population in all four studies. The lowest prevalence was reported among white population, followed by black African population and highest prevalence was reported among South Asian population in all studies. Diabetes prevalence was 1.3- 4 times higher in black Africans than white group. The highest prevalence was recorded

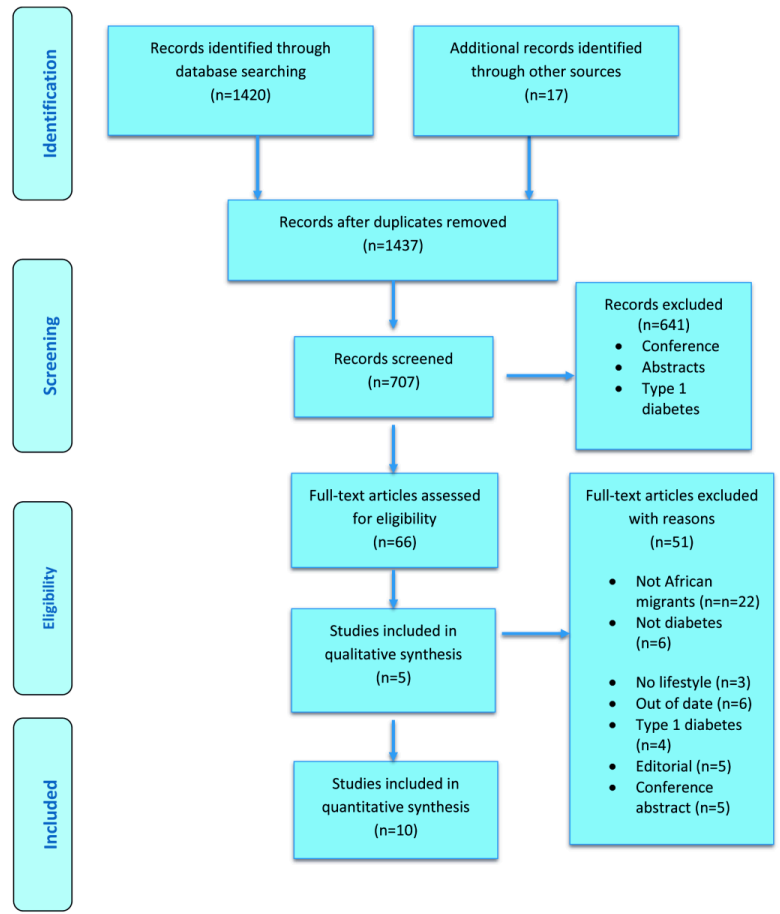

Figure 1

PRISMA flow chart diagram of study selection process in the review.

among South Asians; diabetes was reported to be at least 2.2-7.3 times higher than white population. See Table 5 for diabetes prevalence reported in studies reviewed. 
Table 6. HbA1c outcome measures

\begin{tabular}{|c|c|c|c|c|}
\hline Authors & HbA1c outcome measure & Black Africans & $\begin{array}{l}\text { South } \\
\text { Asians }\end{array}$ & $\begin{array}{l}\text { White } \\
\text { group }\end{array}$ \\
\hline \multirow{5}{*}{ Ballotari et al. $2015^{34}$} & \multirow{5}{*}{ HbA1c > 9\% (\%) } & Male: 27.8 & Male: 29.8 & Male: 12.0 \\
\hline & & Female: 37.5 & & \\
\hline & & Caribbean & & \\
\hline & & Male: 50.0 & & \\
\hline & & Female: 28.6 & $\begin{array}{l}\text { Female: } \\
42.3\end{array}$ & $\begin{array}{l}\text { Female: } \\
12.6\end{array}$ \\
\hline James et al. $2012^{36}$ & $\leq 7.5 \%$ & $49 \%$ & $45 \%$ & $52 \%$ \\
\hline Dreyer et al. $2009^{35}$ & Mean & $8.1 \%$ & $8.0 \%$ & $7.6 \%$ \\
\hline $\begin{array}{l}\text { Fosse-Edorh et al. } \\
2014^{42}\end{array}$ & $\geq 8 \%$ & $30 \%$ & NR & $15 \%$ \\
\hline Weiland et al. $2012^{43}$ & $\leq 7 \%$ & $40.7 \%$ & NR & $53.9 \%$ \\
\hline \multirow{5}{*}{ Biljholt et al. $2018^{47}$} & \multirow{5}{*}{$\leq 7 \%$} & Amsterdam 73.3\% & NR & NR \\
\hline & & Berlin $72.9 \%$ & NR & \\
\hline & & London $66.7 \%$ & NR & \\
\hline & & Urban Ghana 56.3\% & NR & \\
\hline & & Rural Ghana 37.3\% & NR & \\
\hline \multirow[b]{2}{*}{ Choukem et al. $2014^{45}$} & \multirow[b]{2}{*}{ Mean } & Cameroonian 9.9\% & NR & $8.1 \%$ \\
\hline & & $\begin{array}{l}\text { African immigrants } \\
8.6 \%\end{array}$ & & \\
\hline \multirow{4}{*}{ Abubakari et al. $2013^{46}$} & \multirow[b]{2}{*}{ Mean } & $8.33 \%$ & NR & $8.04 \%$ \\
\hline & & $\begin{array}{l}\text { African Caribbean } \\
8.15 \%\end{array}$ & & \\
\hline & \multirow{2}{*}{$\begin{array}{l}\text { Percentage with } \mathrm{HbA} 1 \mathrm{c}>7 \% \\
\text { (\%) }\end{array}$} & 59 & & 57 \\
\hline & & African Caribbean 64 & & \\
\hline \multirow[b]{2}{*}{ Snijder et al. $2017^{40}$} & \multirow{2}{*}{$\begin{array}{l}\text { Percentage with } \mathrm{HbA} 1 \mathrm{c} \leq 7 \% \\
(\%)\end{array}$} & Male: 45.6 & Male: 38.5 & Male: 67.4 \\
\hline & & Female: 54.0 & $\begin{array}{l}\text { Female: } \\
38.2\end{array}$ & $\begin{array}{l}\text { Female: } \\
47.1\end{array}$ \\
\hline \multirow{4}{*}{ Verma et al. $2010^{41}$} & 1997 & 33.6 & 34.1 & 32.5 \\
\hline & 2006 & 77.7 & 75.5 & 77.3 \\
\hline & $\leq 7.0 \%(\%) 1997$ & 3.9 & 4.1 & 0 \\
\hline & $\leq 7.0 \%$ (\%) 2006 & 39.0 & 35.7 & 40.6 \\
\hline
\end{tabular}

HbA1c - haemoglobin A1C, NR - not reported

\section{HAEMOGLOBIN A1C (HBA1C)}

Three papers 35,45,46 reported mean HbA1c differences among all three ethnic groups. All studies reported higher mean HbA1c among black Africans and South Asians than the white population. Although differences were not statistically significant, black Africans had highest mean HbA1c values.

In addition to the mean $\mathrm{HbA1c}$, control $\mathrm{HbA} 1 \mathrm{c}$ was reported among eight studies reviewed. ${ }^{36,40-43,45,47}$ The control target differ among the studies with some studies using target of $\mathrm{HbA1c}<7.0 \% 40,41,43,45,47$, while James et al. ${ }^{36}$ used HbA1c $<7.5 \%$ target and Fosse-Edorh et al. ${ }^{42}$ used HbA1c $<8 \%$ target, Ballotari et al. ${ }^{34}$ reported percentage of participants with $\mathrm{HbA1c}>9 \%$. Most studies reported higher percentage of white population meeting HbA1c target than black African and South Asian populations. ${ }^{34,40 \_36,43}$ In comparison among black African immigrants in different European cities, Biljholt et al. ${ }^{34}$ reported a higher percent- age of HbA1c target met among Ghanaian immigrants in Amsterdam, Berlin while lower percentage was reported in London. Interestingly, the percentage of HbA1c control target was similar in rural Ghana as compared to immigrants in Amsterdam and Berlin while the lowest percentage to meet target was reported in urban Ghana.Table 6 presents detailed HbA1c outcome measures reported.

\section{BLOOD PRESSURE}

Blood pressure measure was reported by five of the studies reviewed. ${ }^{35,41-43,45}$ Blood pressure measure was reported to be higher among black Africans than white and South Asian groups. Similarly, the percentage that met blood pressure target was lowest among black Africans than white and South Asia populations. Although blood pressure target was not significantly different among all ethnic groups in four studies, one study reported a significant difference. Choukem et al. ${ }^{45}$ reported a similar percentage of partic- 
Table 7. Blood pressure outcome measures

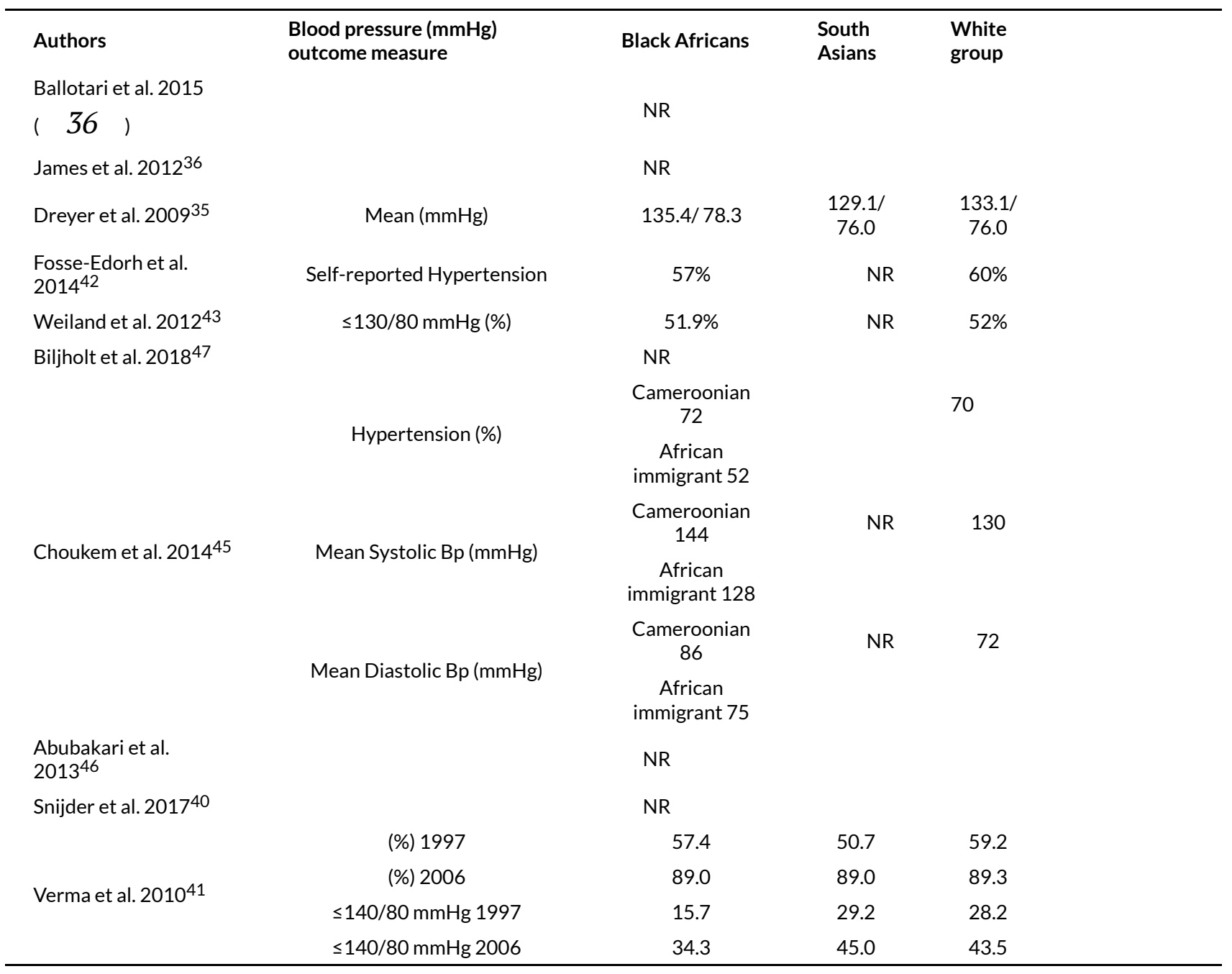

Bp - blood pressure, NR - not reported

ipants with hypertension among Cameroonian and Caucasians respectively, while significantly lower percentage was reported among Cameroonian immigrants (See Table 7 for detailed blood pressure outcome measures reported).

\section{CHOLESTEROL}

Total cholesterol measure was reported by six studies reviewed. ${ }^{35,36,41-43,45}$ Although different cholesterol targets were used in these studies, black Africans were slightly lower in cholesterol target percentage than white and South Asian populations. However, one study reported mean total cholesterol to be lower among Cameroonian immigrants than white groups. ${ }^{45}$ South Asians had the lowest cholesterol level among all ethnic groups. In general, cholesterol measure was not significantly different among all groups.Table 8 presents cholesterol outcome measures as reported in studies reviewed.

\section{BODY MASS INDEX (BMI)}

BMI was reported by four studies in this review. $36,40,45,47$ Three out of the four studies reported lower mean BMI among black Africans than white population. ${ }^{36,45,47}$ Bijlholt et al. ${ }^{47}$ reported lowest BMI among participants in rural Ghana and urban Ghana as compared to immigrants in European sites. Among immigrants in European sites, BMI was similar in Amsterdam and Berlin while highest BMI was reported in London. Choukem et al. ${ }^{45}$ reported similar BMI value among Cameroonian and Cameroon immigrants with the highest BMI reported among Caucasian.

Similarly, James et al. ${ }^{36}$ reported lower BMI among immigrants than the white population. Lowest mean BMI was reported among South Asia, followed by black population while highest BMI was reported among white group. However, Snidjer et al. ${ }^{40}$ was the only study that reported lowest mean BMI among white participants while Ghanaian immigrants were reported to have mean BMI higher than white group.Table 9 presents detailed measure outcomes among quantitative articles reviewed.

\section{QUALITATIVE FINDINGS}

Five qualitative articles were included in this review. ${ }^{37-39,44}$ These articles focus on explaining some factors that may contribute to the management of diabetes among immi- 
Table 8. Cholesterol outcome measures

\begin{tabular}{|c|c|c|c|c|}
\hline Authors & Cholesterol outcome measure & Black Africans & $\begin{array}{l}\text { South } \\
\text { Asians }\end{array}$ & $\begin{array}{l}\text { White } \\
\text { group }\end{array}$ \\
\hline Ballotari et al. $2015^{34}$ & NR & & & \\
\hline James et al. $2012^{36}$ & Total (Mean) & 4.4 & 4.1 & 4.3 \\
\hline Dreyer et al. $2009^{35}$ & Total (Mean) & 4.4 & 4.2 & 4.3 \\
\hline $\begin{array}{l}\text { Fosse-Edorh et al. } \\
2014^{42}\end{array}$ & $\begin{array}{l}\text { Self-reported hypercholesterolemia } \\
\text { (\%) }\end{array}$ & $57 \%$ & & $56 \%$ \\
\hline Weiland et al. $2012^{43}$ & $\mathrm{LDL} \leq 100 \mathrm{mg} / \mathrm{dl}(\%)$ & 53.1 & & $61.3 \%$ \\
\hline Biljholt et al. $2018^{47}$ & NR & & & \\
\hline \multirow[t]{6}{*}{ Choukem et al. $2014^{45}$} & Total cholesterol (mg/dL) & Cameroonians 202 & & 194 \\
\hline & & $\begin{array}{c}\text { African immigrants } \\
185\end{array}$ & & \\
\hline & HDL cholesterol & Cameroonians 40 & & 50 \\
\hline & & $\begin{array}{l}\text { African immigrants } \\
50\end{array}$ & & \\
\hline & LDL cholesterol & Cameroonians 130 & & 116 \\
\hline & & $\begin{array}{c}\text { African immigrants } \\
119\end{array}$ & & \\
\hline Abubakari et al. $2013^{46}$ & NR & & & \\
\hline Snijder et al. $2017^{40}$ & NR & & & \\
\hline \multirow[t]{4}{*}{ Verma et al. $2010^{41}$} & Total (\%) 1997 & 11.6 & 20.1 & 13.3 \\
\hline & Total (\%) 2006 & 80.0 & 77.2 & 82.0 \\
\hline & $\leq 5.0 \mathrm{mmol} / \mathrm{L}(\%) 1997$ & 38.9 & 43.2 & 31.3 \\
\hline & $\leq 5.0 \mathrm{mmol} / \mathrm{L}(\%) 2006$ & 71.9 & 79.2 & 72.6 \\
\hline
\end{tabular}

LDL - low-density lipoprotein, HDL - high-density lipoprotein, NR - not reported

grant population. Some of these factors help to explain the findings of diabetes management from the quantitative articles reviewed.

\section{DELAYED DIAGNOSIS}

Studies reviewed reported how diabetes diagnosis is delayed among participants. Many individuals were reported to know about their diabetes status after visiting hospital for when other health issues. Kahn et al. ${ }^{37}$ reported that participants finding out about having diabetes when in emergency units for other health conditions. Many of the participants were diagnosed with diabetes only after noticing symptoms that made them require me require medication. This was reported to contribute to the management among these individuals, mainly as the late diagnosis can contribute to diabetes complications.

\section{EMOTIONS AT BEING DIAGNOSED}

Emotions at being diagnosed with diabetes were reported to influence the management of diabetes. ${ }^{37,38}$ Participants were reported to be surprised at being diagnosed as it was not expected. Emotions such as denial, anger, acceptance and depression are some of the feelings participants were reported to go through after diagnosis. These emotions have different ways of contributing to the management of diabetes. Denial, anger and depression can be a barrier to proper management of their diabetes as recommended by health-care professionals.

\section{KNOWLEDGE OF CAUSE OF DIABETES}

Many of the participants were reported to have limited knowledge about diabetes as a disease condition. ${ }^{37,38}$ Kandarara et al. ${ }^{38}$ reported how participants explain not to have adequate knowledge about diabetes before diagnosed with the condition. As a result of limited knowledge prior to diagnosis, their management process has been affected. They reported having to learn everything about the condition after diagnosis which makes following management recommendation difficult.

\section{BARRIERS TO MANAGEMENT}

Some factors were reported as barriers to optimal management of diabetes in studies reviewed. Dietary struggle was a factor that was reported by participants as a barrier to their management of diabetes. Another barrier was unfamiliar diets recommended by healthcare professionals in managing diabetes. ${ }^{38,39}$ Difficulty in healthy cooking and cultural influence in dietary habits were also reported as barriers to management. ${ }^{37-39}$ Long hours of working due to the high cost of living were reported to hinder the management of their condition. ${ }^{37}$ Bramberg et al. ${ }^{48}$ used observational method for data collection reported the lack of individualised care by healthcare professionals as barrier to diabetes management. The observational study of diabetes appointment between diabetes nurse and patients reported how patients' concerns on diabetes management were not 
Table 9. BMI outcome measures

\begin{tabular}{|c|c|c|c|c|}
\hline Authors & BMI & Black Africans & $\begin{array}{l}\text { South } \\
\text { Asians }\end{array}$ & $\begin{array}{l}\text { White } \\
\text { group }\end{array}$ \\
\hline Ballotari et al. $2015^{34}$ & & NR & & \\
\hline James et al. $2012^{36}$ & Mean BMI & 30.8 & 27.8 & 33.3 \\
\hline Dreyer et al. $2009^{35}$ & & NR & & \\
\hline $\begin{array}{l}\text { Fosse-Edorh et al. } \\
2014^{42}\end{array}$ & & NR & & \\
\hline \multirow[t]{3}{*}{ Weiland et al. $2012^{43}$} & & NR & & \\
\hline & & Amsterdam 29.7 & NR & NR \\
\hline & & Berlin 29.2 & & \\
\hline \multirow[t]{5}{*}{ Biljholt et al. $2018^{47}$} & $\begin{array}{c}\text { Mean BMI (kg/ } \\
\left.\mathrm{m}^{2}\right)\end{array}$ & London 30.8 & & \\
\hline & & Urban Ghana 27.1 & & \\
\hline & & Rural Ghana 23.9 & & \\
\hline & & Cameroonian 27.9 & NR & 30 \\
\hline & Mean BMI & $\begin{array}{c}\text { African immigrant } \\
27.4\end{array}$ & & \\
\hline \multirow[t]{4}{*}{ Choukem et al. $2014^{45}$} & \multirow{2}{*}{$\begin{array}{c}\mathrm{BMI} \geq \underset{(\%)}{25 \mathrm{~kg} / \mathrm{m}^{2}} \\
\end{array}$} & Cameroonian 74.0 & & 81.4 \\
\hline & & $\begin{array}{c}\text { African immigrant } \\
68.4\end{array}$ & & \\
\hline & \multirow{2}{*}{$\begin{array}{c}\mathrm{BMI} \geq 30 \mathrm{~kg} / \mathrm{m}^{2} \\
(\%)\end{array}$} & Cameroonian 32.0 & & 44.2 \\
\hline & & $\begin{array}{c}\text { African immigrant } \\
24.5\end{array}$ & & \\
\hline \multirow[t]{2}{*}{ Abubakari et al. $2013^{46}$} & Mean BMI & 31.35 & 31.43 & 33.44 \\
\hline & & Male: 26.7 & Male: 25.8 & Male: 25.2 \\
\hline Snijder et al. $2017^{40}$ & Mean BMI & Female: 29.6 & $\begin{array}{c}\text { Female: } \\
26.7\end{array}$ & $\begin{array}{l}\text { Female: } \\
24.4\end{array}$ \\
\hline Verma et al. $2010^{41}$ & & NR & & \\
\hline
\end{tabular}

BMI - body mass index, NR - not reported

properly addressed due to the lack of individualised care for these patients.

\section{FACILITATORS TO MANAGEMENT}

Studies also reported some factors that enhance better management of their diabetes. ${ }^{38,44}$ This was reported by participants to help in managing their diabetes. Social support and having consistent routines or everyday practices to follow in managing diabetes was reported to enhance their diabetes management. ${ }^{38,44}$ Having culturally tailored recommended dietary plans was reported to facilitate diabetes management among participants. ${ }^{39}$ There are important implications of the findings from this review that requires further discussions.

\section{DISCUSSION}

This review was conducted to explore management outcomes differences among black Africans, South Asians and white population. This review found poorer diabetes management outcomes among black Africans as compared to South Asia and white population. Diabetes prevalence was higher among black Africans than the white population in all studies reviewed. In addition, diabetes management outcomes like HbA1c, blood pressure and cholesterol level were slightly higher among black Africans than other groups. Blood glucose level using HbA1c outcome was slightly higher among black Africans while white population had the lowest measure.

Black African participants in all studies were less likely to meet blood pressure target than white and South Asian populations except for one study where immigrants had lower blood pressure than Caucasians. ${ }^{45}$ It is worthy to note that these studies use different targets for all outcomes measured which might influence their findings. Studies conducted in the UK used stricter target measures of 130/ $80 \mathrm{mmHg}$ than the $140 / 90 \mathrm{mmHg}$ used in studies conducted in the USA. It is important to note that recently, blood pressure classification for hypertension have been moved from $140 / 90 \mathrm{mmHg}$ to $130 / 80 \mathrm{mmHg}$ which will make more than $46 \%$ of American hypertensive. ${ }^{63}$ New studies after this review may use the stricter target than studies in this review which may affect their findings.

In general, black Africans were reported to be least likely to meet all three outcome targets, although lower BMI was reported in three of the four studies that reported BMI measure. This review shows diabetes management disparity among ethnic minority groups when compared to white population; this disparity is particularly pronounced among black Africans. Similar findings have been reported in reviews. ${ }^{64,65}$ Agyemang et al. ${ }^{66}$ showed that blood pressure 
increase among African Caribbean population with increasing age exceeding other ethnic groups like white population.

Lower likelihood of meeting all HbA1c, blood pressure and cholesterol levels have been reported to increase the risk of developing diabetes complications. ${ }^{67}$

Another interesting finding is the differences in immigrant management on different European sites as reported in the review. London was a site that reported significantly lower management outcomes among other European sites although higher education percentage was reported among African immigrants from this site. This shows that there is poorer management outcome among immigrants, vary with country residence. It is therefore important to look into environmental influence on diabetes management.

Looking into diabetes management, some explanations for poorer management among black Africans were reported among the qualitative studies reviewed. These include the late diagnosis of diabetes, dietary struggles, and lack of individualised care. These factors are important in contributing to the adequate management of diabetes as they relate to lifestyle factors in the management of diabetes. ${ }^{68}$ Lifestyle factors have been shown to greatly influence the successful management of diabetes. ${ }^{69}$ This is particularly as diabetes management is importantly a selfmanagement condition. ${ }^{57}$ Another explanation for the poorer management among black Africans might be due to lower awareness about diabetes. Lower awareness has been reported among this group concerning diabetes. ${ }^{70}$ Many of whom do not know their diabetes status and limited knowledge about diabetes as a condition was reported in the qualitative studies reviewed.

From the studies conducted in the UK in this review, only two studies reported separate diabetes management among black African and African Caribbean. ${ }^{34,46}$ This highlights the need to study these groups separately rather than combining all African groups as one homogeneous group. It has already been recommended to explore cardiovascular disease risk factors and health behaviours by country of emigration. ${ }^{71}$ This is of particular importance as it was not possible to know the percentage of black Africans and African Caribbean, which makes up "blacks" as reported in some studies. This makes interpreting the findings from these studies to each group difficult. In terms of the black populations, it is known that African Caribbean has a higher population and higher integration into the UK society due to the long years of stay than black Africans making them less represented in health research. ${ }^{22}$ However, black Africans are among the fastest growing ethnic groups in the UK 72,73 This should be reflected in studies that are conducted among "blacks".

The variation in study methodology, measurement method, outcome target measures and the combination of black African and African Caribbean populations in some study should be put into consideration when interpreting the findings of this review. Standardizing the target measures for diabetes management outcome will be valuable to future research. In addition, there is a need for meta-analysis among African Caribbean and black African due to the difference among these groups. Better exploration of diabetes outcome among African immigrants in various loca- tions in Europe is needed to understanding diabetes management as differences noted in this review. More research needed among black Africans residence outside London as this was the study location used in most studies conducted in the UK.

\section{LIMITATIONS}

Since the early 2000s, several reviews have been conducted to explore health differences among ethnic minority groups in western countries. ${ }^{23-25}$ However, no review has focused on comparison of health outcomes among ethnic groups living with diabetes with particular focus on black Africans. This therefore presents the findings of the review to be unique and relevant in terms of diabetes management among ethnic groups. However, several limitations were noted in conducting this review.

Limitations of this review include the small number of studies that were reviewed as relevant to the study aim. This reflected the limited literature available for comparison of diabetes management outcomes among black African, South Asian and white populations. Studies varied in terms of the research goal with only four studies having the primary goal of diabetes management outcome as primary aim of the studies. ${ }^{34,36,41,45}$ This in effect can influence the findings of this review as this highlights the lack of comparison studies among these three population groups.

In this review several decisions were made that might have affected the retrieval of studies that were included in the review. One of such decisions is the year of publication criteria that was used to retrieve only articles published after 2006. This was done to ensure that articles that have current information are included in the review. In addition, only peer review articles were included to reduce retrieval of low quality articles. Finally, only articles published in English were included in the review; this might have the effect of excluding relevant articles written in other languages. However, this was managed by ensuring that all articles were searched and relevant articles retrieved before exclusion based on language limiters. Abstracts of articles that have potential to be included in the review were translated before decision to include or exclude. However, none of the articles written in other languages was found to be relevant for inclusion in this review.

\section{CONCLUSION}

Diabetes prevalence is higher among black Africans than the white population. In addition, poorer management outcomes was observed among this population compared to both white and South Asian populations as noted in the reported biomedical outcomes (HbA1c, blood pressure, cholesterol). This disparity in diabetes management needs to be addressed to narrow the inequality gap among ethnic groups. Due to different targets used in the studies review, it was not possible to perform a meta-analysis. Standardised target measure is needed in future research to better use findings from studies. In terms of black group, there is need to for future research to recognise the different subsets of black population to allow findings tailored to a particular group in this population. 


\section{ACKNOWLEDGEMENTS}

Many thanks to Bournemouth University's Department of Public Health and Prof Edwin Van Teijlingen for their support.

\section{FUNDING}

This is part of a $\mathrm{PhD}$ project funded through Bournemouth University VC Scholarship.

\section{COMPETING INTERESTS}

The authors have completed the Unified Competing Interest form at http://www.icmje.org/coi disclosure.pdf (available on request from the corresponding author) and declare no conflict of interest.

\section{CORRESPONDENCE TO:}

Folashade Alloh

Department of Human Sciences \& Public Health

Faculty of Health and Social Sciences

Bournemouth University

Bournemouth House

Christchurch Road

BH1 1RX, UK

falloh@bournemouth.ac.uk 


\section{REFERENCES}

1. World Health Organization. Diabetes Factsheet. Published online 2018. Accessed May 20, 2019. http s://www.who.int/news-room/fact-sheets/detail/diabet es

2. World Health Organization. Global Report on Diabetes. Published online 2016. Accessed June 3, 2018. https://apps.who.int/iris/bitstream/handle/1066 5/204871/9789241565257_eng.pdf?sequence $=1$

3. Whiting DR, Guariguata L, Weil C, Shaw J. IDF diabetes atlas: global estimates of the prevalence of diabetes for 2011 and 2030. Diabetes Res Clin Pract. 2011;94(3):311-321. doi:10.1016/i.diabres.2011.10.02 $\underline{9}$

4. Guariguata L, Whiting DR, Hambleton I, Beagley J, Linnenkamp U, Shaw JE. Global estimates of diabetes prevalence for 2013 and projections for 2035. Diabetes Res Clin Pract. 2014;103(2):137-149. doi:10.1016/i.dia bres.2013.11.002

5. Ogurtsova K, da Rocha Fernandes JD, Huang Y, et al. IDF Diabetes Atlas: Global estimates for the prevalence of diabetes for 2015 and 2040. Diabetes Res Clin Pract. 2017;128:40-50. doi:10.1016/j.diabre s.2017.03.024

6. Hu FB. Globalization of diabetes: the role of diet, lifestyle, and genes. Diabetes Care.

2011;34(6):1249-1257. doi:10.2337/dc11-0442

7. Diabetes UK. Facts and Statistics. Published online 2016. Accessed April 28, 2018. https://www.diabetes.o rg.uk/Documents/Position\%20statements/DiabetesU K Facts_Stats_Oct16.pdf

8. Centers for Disease Control. National Diabetes Statistics Report, 2017: Estimates of Diabetes and Its Burden in the United States. Published online 2017. Accessed May 19, 2018. https://www.cdc.gov/diabete s/pdfs/data/statistics/national-diabetes-statistics-rep ort.pdf

9. Rosella LC, Lebenbaum M, Fitzpatrick T, Zuk A, Booth GL. Prevalence of prediabetes and undiagnosed diabetes in Canada (2007-2011) according to fasting plasma glucose and HbA1c screening criteria. Dia Care. 2015;38(7):1299-1305. doi:10.2337/dc14-2474

10. Diabetes Charter for Canada. Diabetes in canada. Published online 2016. Accessed May 13, 2018. http s://www.diabetes.ca/getmedia/513a0f6c-b1c9-4e56-a 77c-6a492bf7350f/diabetes-charter-backgrounder-nat ional-english.pdf.aspx
11. Creatore MI, Moineddin R, Booth G, et al. Ageand sex-related prevalence of diabetes mellitus among immigrants to Ontario, Canada. CMAJ. 2010;182(8):781-789. doi:10.1503/cmaj.091551

12. Moss M, Hawthorne K, Hughes N, Lavelle D, Tan MC. Working and engaging with minority ethnic communities in an urban setting. J Diabetes Nurs. 2008;12:310-338.

13. National Institute for Health and Care Excellence. Type 2 diabetes: prevention in people at high risk. Published online 2012. Accessed April 10, 2018. http s://www.nice.org.uk/guidance/ph38/resources/typ e-2-diabetes-prevention-in-people-at-high-risk-1996 $\underline{304192197}$

14. Zagefka H. Ethnicity, Concepts of. The Wiley Blackwell Encyclopedia of Race, Ethnicity, and Nationalism. Published online December 30, 2015:1-3. doi:10.1002/9781118663202.wberen428

15. Luckett T, Goldstein D, Butow PN, et al. Psychological morbidity and quality of life of ethnic minority patients with cancer: a systematic review and meta-analysis. Lancet Oncol. 2011;12(13):1240-1248. doi:10.1016/s1470-2045(11)7 $\underline{0212-1}$

16. Oldroyd J, Banerjee M, Heald A, Cruickshank K. Diabetes and ethnic minorities. Postgrad Med J. 2005;81(958):486-490. doi:10.1136/pgmj.2004.029124

17. Montesi L, Caletti MT, Marchesini G. Diabetes in migrants and ethnic minorities in a changing World. World J Diabetes. 2016;7(3):34. doi:10.4239/wjd.v7.i $\underline{3.34}$

18. Meeks KAC, Stronks K, Beune EJAJ, et al. Prevalence of type 2 diabetes and its association with measures of body composition among African residents in the Netherlands - The HELIUS study. Diabetes Res Clin Pract. 2015;110(2):137-146. doi:10.1 016/j.diabres.2015.09.017

19. Bleich SN, Jarlenski MP, Bell CN, LaVeist TA. Health inequalities: trends, progress, and policy. Annu Rev Public Health. 2012;33(1):7-40. doi:10.1146/ annurev-publhealth-031811-124658

20. Reuven Y, Dreiher J, Shvartzman P. The prevalence of diabetes, hypertension and obesity among immigrants from East Africa and the former Soviet Union: a retrospective comparative 30 -year cohort study. Cardiovasc Diabetol. 2016;15(1):74. doi:10.118 6/s12933-016-0392-7 
21. Office of National Statistics. Ethnicity and National Identity in England and Wales 2011. Published online 2012. Accessed September 30, 2018. https://www.ons.gov.uk/peoplepopulationandcommu nity/culturalidentity/ethnicity/articles/ethnicityandn ationalidentityinenglandandwales/2012-12-11

22. Goff LM, Timbers L, Style H, Knight A. Dietary intake in Black British adults; an observational assessment of nutritional composition and the role of traditional foods in UK Caribbean and West African diets. Public Health Nutr. 2014;18(12):2191-2201. do $\mathrm{i}: 10.1017 / \mathrm{s} 1368980014002584$

23. Johnson M, Everson-Hock E, Jones R, Woods HB, Payne N, Goyder E. What are the barriers to primary prevention of type 2 diabetes in black and minority ethnic groups in the UK? A qualitative evidence synthesis. Diabetes Res Clin Pract. 2011;93(2):150-158. doi:10.1016/j.diabres.2011.06.00 4

24. Wilson C, Alam R, Latif S, Knighting K, Williamson S, Beaver K. Patient access to healthcare services and optimisation of self-management for ethnic minority populations living with diabetes: a systematic review. Health Soc Care Community. 2012;20(1):1-19. doi:10.1111/j.1365-2524.2011.0101 7.x

25. Zeh P, Sandhu HK, Cannaby AM, Sturt JA. The impact of culturally competent diabetes care interventions for improving diabetes-related outcomes in ethnic minority groups: a systematic review. Diabet Med. 2012;29(10):1237-1252. doi:10.11 11/j.1464-5491.2012.03701.x

26. Grant MJ, Booth A. A typology of reviews: an analysis of 14 review types and associated methodologies. Health Info Libr J. 2009;26(2):91-108. doi:10.1111/j.1471-1842.2009.00848.x

27. Hewitt-Taylor J. The Essential Guide to Doing a Health and Social Care Literature Review. Routledge; 2017. doi:10.4324/9781315643472

28. Booth A, Sutton A, Papaioannou D. Systematic approaches to a successful literature review: Sage London. Published online 2016.

29. Critical Appraisal Skill Programme Tools. CASP Checklists [online]. Published 2014. Accessed June 16, 2018. http://www.casp-uk.net/\#!casp-tools-checklist s/c18f8

30. Smith J, Noble H. Reviewing the literature. Evid Based Nurs. 2016;19:2-3. doi:10.1136/eb-2015-10225 $\underline{2}$
31. Noble H, Smith J. Reviewing the literature: choosing a review design. Evid Based Nurs. 2018;21(2):39-41. doi:10.1136/eb-2018-102895

32. Farrance C, Tsofliou F, Clark C. Adherence to community based group exercise interventions for older people: A mixed-methods systematic review. Prev Med. 2016;87:155-166. doi:10.1016/j.ypmed.201 $\underline{6.02 .037}$

33. Elamin MB, Flynn DN, Bassler D, et al. Choice of data extraction tools for systematic reviews depends on resources and review complexity. J Clin Epidemiol. 2009;62(5):506-510. doi:10.1016/i.jclinepi.2008.10.01 6

34. Ballotari P, Caroli S, Ferrari F, et al. Differences in diabetes prevalence and inequalities in disease management and glycaemic control by immigrant status: a population-based study (Italy). BMC Public Health. 2015;15(1). doi:10.1186/s12889-015-1403-4

35. Dreyer G, Hull S, Aitken Z, Chesser A, Yaqoob MM. The effect of ethnicity on the prevalence of diabetes and associated chronic kidney disease. OJM. 2009;102(4):261-269. doi:10.1093/qjmed/hcn177

36. James GD, Baker P, Badrick E, Mathur R, Hull S, Robson J. Ethnic and social disparity in glycaemic control in type 2 diabetes; cohort study in general practice 2004-9. J R Soc Med. 2012;105(7):300-308. do i:10.1258/jrsm.2012.110289

37. Kahn LS, Vest BM, Karl R, et al. Living with diabetes on buffalo, New York's culturally diverse West Side. Chronic Illn. 2013;9(1):43-56. doi:10.1177/ 1742395312450895

38. Kindarara DM, McEwen MM, Crist JD, Loescher LJ. Health-Illness Transition Experiences With Type 2 Diabetes Self-management of Sub-Saharan African Immigrants in the United States. Diabetes Educ. 2017;43(5):506-518. doi:10.1177/0145721717724725

39. Kohinor MJE, Stronks K, Nicolaou M, Haafkens JA. Considerations affecting dietary behaviour of immigrants with type 2 diabetes: A qualitative study among Surinamese in the Netherlands. Ethn Health. 2011;16(3):245-258. doi:10.1080/13557858.2011.5635 $\underline{57}$

40. Snijder MB, Agyemang C, Peters RJ, Stronks K, Ujcic-Voortman JK, van Valkengoed IGM. Case Finding and Medical Treatment of Type 2 Diabetes among Different Ethnic Minority Groups: The HELIUS Study. J Diabetes Res. 2017;2017:1-8. doi:10.1155/201 7/9896849 
41. Verma A, Birger R, Bhatt H, et al. Ethnic disparities in diabetes management: a 10-year population-based repeated cross-sectional study in UK primary care. J Public Health (Oxf).

2010;32(2):250-258. doi:10.1093/pubmed/fdp114

42. Fosse-Edorh S, Fagot-Campagna A, Detournay B, et al. Type 2 diabetes prevalence, health status and quality of care among the North African immigrant population living in France. Diabetes Metab. 2014;40(2):143-150. doi:10.1016/i.diabet.2013.11.005

43. Wieland ML, Morrison TB, Cha SS, Rahman AS, Chaudhry R. Diabetes Care Among Somali Immigrants and Refugees. J Community Health. 2012;37(3):680-684. doi:10.1007/s10900-011-9499-7

44. Wallin AM, Löfvander M, Ahlström G. Diabetes: A cross-cultural interview study of immigrants from Somalia. J Clin Nurs. 2007;16(11c):305-314. doi:10.11 11/j.1365-2702.2007.02099.x

45. Choukem SP, Fabreguettes C, Akwo E, et al. Influence of migration on characteristics of type 2 diabetes in sub-Saharan Africans. Diabetes Metab. 2014;40(1):56-60. doi:10.1016/j.diabet.2013.07.004

46. Abubakari AR, Jones M, Lauder W, Kirk A, Anderson J, Naderali E. Ethnic differences and sociodemographic predictors of illness perceptions, selfmanagement, and metabolic control of type 2 diabetes. Int J Gen Med. Published online July 2013:617. doi:10.2147/iigm.s46649

47. Bijlholt M, Meeks KAC, Beune E, et al. Type 2 diabetes mellitus management among Ghanaian migrants resident in three European countries and their compatriots in rural and urban Ghana - The RODAM study. Diabetes Res Clin Pract. 2018;136:32-38. doi:10.1016/j.diabres.2017.11.032

48. Brämberg EB, Dahlborg-Lyckhage E, Määttä S. Lack of individualized perspective: A qualitative study of diabetes care for immigrants in Sweden. Nurs Health Sci. 2012;14(2):244-249. doi:10.1111/j.1442-20 18.2012.00684.x

49. O’Connor MY, Thoreson CK, Ricks M, et al. Worse cardiometabolic health in African immigrant men than African American men: reconsideration of the healthy immigrant effect. Metab Syndr Relat Disord. 2014;12(6):347-353. doi:10.1089/met.2014.0026

50. Mitri J, Gabbay RA. Measuring the quality of diabetes care. Am J Manag Care. 2016;22:SP147.

51. International Diabetes Federation.

Recommendations for Managing Type 2 diabetes in Primary Care. Published online 2017. Accessed June 17, 2018. https://www.idf.org/managing-type2-diabet es
52. World Health Organization. Prevention of Cardiovascular disease: Guidelines for assessment and management of cardiovascular risk. Published online 2007. Accessed July 18, 2018. https://apps.wh o.int/iris/bitstream/handle/10665/43685/9789241547 178 eng.pdf? sequence $=1$ \&isAllowed $=y$

53. National Institute for Health and Care Excellence. Blood glucose management: HbA1c measurement and targets. Published online 2015. Accessed March 22, 2018. https://www.nice.org.uk/guidance/ng28/chapte $\underline{\mathrm{r} / 1-\text { recommendations }}$

54. American Diabetes Association. Glycemic targets. Published 2016. Accessed May 28, 2018. http://care.di abetesjournals.org/content/39/Supplement_1/S39

55. National Institute for Health and Care Excellence. Blood Pressure Measurement. Published online 2009. Accessed May 21, 2018. https://www.nice.org.uk/guid ance/ng28/chapter/1-recommendations

56. de Boer IH, Bangalore S, Benetos A, et al. Diabetes and hypertension: a position statement by the American Diabetes Association. Dia Care. 2017;40(9):1273-1284. doi:10.2337/dci17-0026

57. National Institute for Health and Care Excellence. Majority of people with diabetes 'not meeting cholesterol targets'. Published online 2012. Accessed March 22, 2018. https://www.nice.org.uk/news/articl e/majority-of-people-with-diabetes-not-meeting-cho lesterol-targets

58. Eldor R, Raz I. American Diabetes Association indications for statins in diabetes: is there evidence? Diabetes Care. 2009;32(suppl_2):S384-S391. doi:10.23 37/dc09-s345

59. Aveyard H. Doing a Literature Review in Health and Social Care: A Practical Guide. McGraw-Hill Education (UK); 2014.

60. Abubakari AR, Jones MC, Lauder W, Kirk A, Anderson J, Devendra D. Associations between knowledge, illness perceptions, self-management and metabolic control of type 2 diabetes among African and European-origin patients. J Nurs Healthc Chronic Illn. 2011;3(3):245-256. doi:10.1111/j.1752-9824.201 1.01098.x

61. Agyemang C. Negro, Black, Black African, African Caribbean, African American or what? Labelling African origin populations in the health arena in the 21st century. J Epidemiol Community Health. 2005;59(12):1014-1018. doi:10.1136/jech.2005.03596 $\underline{4}$ 
62. Commodore-Mensah Y, Himmelfarb CD, Agyemang C, Sumner AE. Cardiometabolic Health in African Immigrants to the United States: A Call to Reexamine Research on African-descent populations. Ethn Dis. 2015;25(3):373-380. doi:10.18865/ed.25.3.3 $\underline{73}$

63. de Boer IH, Bakris G, Cannon CP. Individualizing blood pressure targets for people with diabetes and hypertension: Comparing the ada and the acc/aha recommendations. JAMA. 2018;319(13):1319-1320. $\underline{\mathrm{d}}$ oi:10.1001/jama.2018.0642

64. Agyemang C, Bhopal RS. Is the blood pressure of South Asian adults in the UK higher or lower than that in European white adults? A review of crosssectional data. J Hum Hypertens. 2002;16(11):739-751. doi:10.1038/sj.jhh.1001488

65. Agyemang C, Bhopal R. Is the blood pressure of people from African origin adults in the UK higher or lower than that in European origin white people? A review of cross-sectional data. J Hum Hypertens. 2003;17(8):523-534. doi:10.1038/sj.jhh.1001586

66. Agyemang C, Humphry RW, Bhopal R. Divergence with age in blood pressure in African-Caribbean and white populations in England: Implications for screening for hypertension. Am J Hypertens. 2012;25(1):89-96. doi:10.1038/ajh.2011.160

67. Holman RR, Paul SK, Bethel MA, Matthews DR, Neil HAW. 10-year follow-up of intensive glucose control in type 2 diabetes. $N$ Engl J Med.

2008;359(15):1577-1589. doi:10.1056/nejmoa0806470
68. Tenkorang EY. Early onset of type 2 diabetes among visible minority and immigrant populations in Canada. Ethn Health. 2016;22(3):266-284. doi:10.108 $\underline{0 / 13557858.2016 .1244623}$

69. Diabetes Prevention Program Research Group. Reduction in the incidence of type 2 diabetes with lifestyle intervention or metformin. $N$ Engl J Med. 2002;346:393-403. doi:10.1056/NEJMoa01251211832 $\underline{527}$

70. Ilunga Tshiswaka D, Ibe-Lamberts KD, Mulunda DM, Iwelunmor J. Perceptions of Dietary Habits and Risk for Type 2 Diabetes among Congolese Immigrants. J Diabetes Res. 2017;2017:1-8. doi:10.115 $\underline{5 / 2017 / 4736176}$

71. Sewali B, Harcourt N, Everson-Rose SA, et al. Prevalence of cardiovascular risk factors across six African Immigrant Groups in Minnesota. BMC Public Health. 2015;15(1). doi:10.1186/s12889-015-1740-3

72. Office of National Statistics. Census gives insights into characteristics of London's population. Published online 2011. Accessed January 30, 2018. htt ps://webarchive.nationalarchives.gov.uk/2016010812 5005/http://www.ons.gov.uk/ons/rel/mro/news-releas e/census-2-1----london/census-gives-insights-into-c haracteristics-of-london-s-population.html

73. Sunak R, Rajeswaran S. A portrait of modern Britain: Policy Exchange London. Published online 2014. 